\title{
Design and Profit Allocation in Two-Echelon Heterogeneous Cooperative Logistics Network Optimization
}

\author{
Yong Wang $\mathbb{D},{ }^{1,2}$ Yingying Yuan, ${ }^{1}$ Kevin Assogba $\mathbb{D},{ }^{3}$ Ke Gong $\mathbb{D},{ }^{1}$ Haizhong Wang, \\ Maozeng $X u,{ }^{1}$ and Yinhai Wang ${ }^{4,5}$ \\ ${ }^{1}$ School of Economics and Management, Chongqing Jiaotong University, Chongqing 400074, China \\ ${ }^{2}$ School of Management and Economics, University of Electronic Science and Technology, Chengdu 610054, China \\ ${ }^{3}$ School of Civil and Construction Engineering, Oregon State University, Corvallis, OR 97330, USA \\ ${ }^{4}$ Department of Civil and Environmental Engineering, University of Washington, Seattle, WA 98195-2700, USA \\ ${ }^{5}$ Transportation Data Science Research Center, College of Transportation Engineering, Tongji University, Shanghai 201804, China
}

Correspondence should be addressed to Yong Wang; yongwx6@gmail.com,

Kevin Assogba; kevinassogba@gmail.com, and Ke Gong; gks_cn@163.com

Received 31 December 2017; Revised 21 March 2018; Accepted 8 April 2018; Published 16 May 2018

Academic Editor: Ángel Ibeas

Copyright (C) 2018 Yong Wang et al. This is an open access article distributed under the Creative Commons Attribution License, which permits unrestricted use, distribution, and reproduction in any medium, provided the original work is properly cited.

In modern supply chain, logistics companies usually operate individually and optimization researches often concentrate on solving problems related to separate networks. Consequences like the complexity of urban transportation networks and long distance deliveries or pickups and pollution are leading problems to more expenses and more complaints from environment protection organizations. A solution approach to these issues is proposed in this article and consists in the adoption of two-echelon heterogeneous cooperative logistics networks (THCLN). The optimization methodology includes the formation of cooperative coalitions, the reallocation of customers to appropriate logistics facilities, and the determination of the best profit allocation scheme. First, a mixed integer linear programing model is introduced to minimize the total operating cost of nonempty coalitions. Thus, the Genetic Algorithm (GA) and the Particle Swarm Optimization (PSO) algorithm are hybridized to propose GA-PSO heuristics. GAPSO is employed to provide good solutions to customer clustering units' reallocation problem. In addition, a negotiation process is established based on logistics centers as coordinators. The case study of Chongqing city is conducted to verify the feasibility of THCLN in practice. The grand coalition and two heterogeneous subcoalitions are designed, and the collective profit is distributed based on cooperative game theory. The Minimum Cost Remaining Savings (MCRS) model is used to determine good allocation schemes and strictly monotonic path principles are considered to evaluate and decide the most appropriate coalition sequence. Comparisons proved the combination of GA-PSO and MCRS better as results are found closest to the core center. Therefore, the proposed approach can be implemented in real world environment, increase the reliability of urban logistics network, and allow decision makers to improve service efficiency.

\section{Introduction}

Cooperative game theory consists in the formation of coalitions and the effective allocation of the collective payoff to each participant. In modern supply chain, coalitions can be made up of facilities from either the same level or different echelons. Therefore, in a network comprising logistics centers (LCs), Distribution Centers (DCs), Pickup Centers (PCs), and customers, coalitions are generally formed vertically with LCs, DCs, and PCs or horizontally among DCs or PCs. A
Two-echelon Heterogeneous Cooperative Logistics Network (THCLN) is a collaborative network with vertical synergies among LCs, DCs, and PCs, homogeneous horizontal synergies among DCs or PCs, and heterogeneous synergies among LCs and DCs or PCs. Properly optimizing THCLN can not only enhance the operational efficiency and avoid waste of resources, but also maximize players' profits. In addition, the implementation of a coordinated transportation system can contribute to achieve more stable coalitions [1]. However, well-developed negotiation mechanisms and a fair allocation 
of payoffs are necessary to increase players' willingness to participate [2].

The optimization of THCLN is a complex process since multiple customers, transportation resources, and profit allocations problems are combined to enhance practicability. Previous researches have focused on establishing a mixed integer programming model and designing efficient algorithms for similar optimization purposes. But due to the number of customers, evolutionary algorithms are usually confronted to problems modeling the amount of available data. Thus, a commonly used approach consists in clustering customers into small units before the application of intelligent algorithms $[3,4]$. Clustering can also facilitate the management of companies' activities and allow decision makers to obtain more effective results by assigning customer clustering units to suitable suppliers $[5,6]$.

Solving customer clustering units' assignment problem is a basic network optimization problem and first appeared as the Quadratic Assignment Problem (QAP) in Dantzig et al. (1959). The standard assignment problem is a traditional binary programming problem usually solved by exact methods. However, as the number of tasks had increased, QAPs became more complicated to solve. Therefore, researchers like Li et al. [7] concentrated on applying heuristic algorithms to assess large-scale location and layout problems. Recently, Sghir et al. [8] proposed an optimization method based on intelligent multiple agents to solve QAPs and provided the algorithm with the capability of learning and dynamically selecting agents. The efficiency of QAP solutions facilitates the optimization of vehicle routing problems or any logistics transportation-related subject. In other words, the reassignment of customers units not only does have significant impacts on the configuration of the network but also influences future decisions.

With the rising need of intelligent approaches for logistics network optimization, heuristic algorithms like the Particle Swarm Optimization (PSO) and the Genetic Algorithm (GA) have been successfully applied in many domains $[9,10]$. Shimizu and Miura [11] proposed a multipopulation based discrete PSO algorithm in order to handle binary decision variables and optimize large-scale logistics networks. Cupić and Teodorović [12] proposed an integer programing model to maximize companies' total profits and service quality and employed the Genetic Algorithm to solve a multiobjective hub location-allocation problem. Haddar et al. [13] proposed a 0-1 mixed interlinear programming model and combined the quantum PSO with an iterative heuristic algorithm to solve the Knapsack sharing problem. Guo et al. [14] used GA and PSO to study forward and reverse flows of fresh food supply chains in Shanghai and verified the validity of the model under low-carbon emission environments. Wang et al. $[15,16]$ combined an improved ant colony algorithm with GA to optimize networks through the reassignment of customers to proper logistics facilities. Alshamsi and Diabat [17] introduced a GA capable of solving complex problems with thousands continuous and binary variables, constraints, and multiple integer variables. The proposed algorithm was applied and proved many times faster than GAMS. Atashpendar et al. [18] extended the speed-constrained multiobjective
PSO algorithm to increase computation speed, convergence, and solution quality.

In logistics network cooperation, game theoretic methods are usually applied to model participants' behaviors in different scenarios and evaluate their contribution to the coalition as well as the proportion of allocated profits $[19,20]$. Hernández and Peeta [21] evaluated the influences of collaboration mechanism on carriers and explored the differences with noncollaborative environments. Findings expressed high degree collaboration as beneficial to increase participants' profits. Guajardo and Rönnqvist [22] addressed the collaborative logistics network optimization problem and studied coalitions' layouts under core stability and equilibrium constraints. Applications in the forest transportation domain and for oil operations have been used to verify the relevance of the proposed optimization model. Wang et al. $[15,16]$ introduced collaboration as means of optimizing a single echelon multiple centers vehicle routing problem and applied an improved version of the Shapley value model to allocate profit.

Extensive articles have solved variants of network optimization problems, but there are still some shortcomings in the literature. (a) Researchers had significant achievements in the optimization of multicenter logistics network. However, few articles studied networks where PCs and DCs can independently collaborate with LCs. (b) The design of cooperative networks with different types of logistics facilities has not been studied particularly from aspects like coalitions' homogeneity or heterogeneity. (c) Collaboration among facilities of the same coalitions should incorporate sharing of transportation resources in order to yield more cost savings.

To alleviate computational complexity, our paper will first examine similar characteristics of customers and cluster them into groups. Therefore, large logistics areas will be divided into smaller and easily manageable units to be further assigned to distribution or pickup centers. For the allocation of customer clustering units and vehicles, we combine Genetic Algorithm (GA) and Particle Swarm Optimization (PSO) algorithm to increase solution efficiency. In order to achieve stability, the consultative process among the participants is coordinated by logistics centers owing to their capability at performing distribution and collection activities. In such a position, LCs could be more influential and better coordinate DCs and PCs. Furthermore, we compute the collective payoff of each coalition and propose a profit-sharing mechanism using the Minimum Cost Remaining Savings (MCRS) model to ensure a fair distribution. Before the formation of a coalition, DCs and PCs used to independently operate at the second echelon of the logistics network and caused the unreasonable exploitation of resources, half loaded vehicles, and the increase of traffic density. By including the sharing transportation fleets in collaboration's contracts, companies would significantly save cost and rationally use available resources. In addition, an effective customer clustering units' assignment procedure should reduce the complexity of the logistics network and make the optimization process conducive to obtaining more efficient customer assignment results and fair profit distribution. 


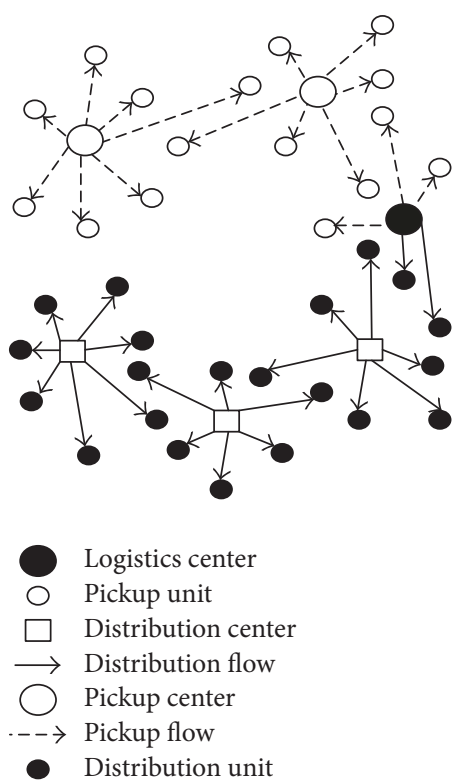

(a) Pickup and delivery before logistics network optimization

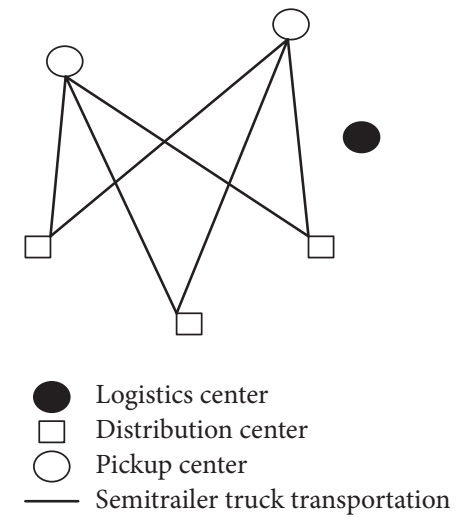

(b) Product flow among facilities before logistics network optimization
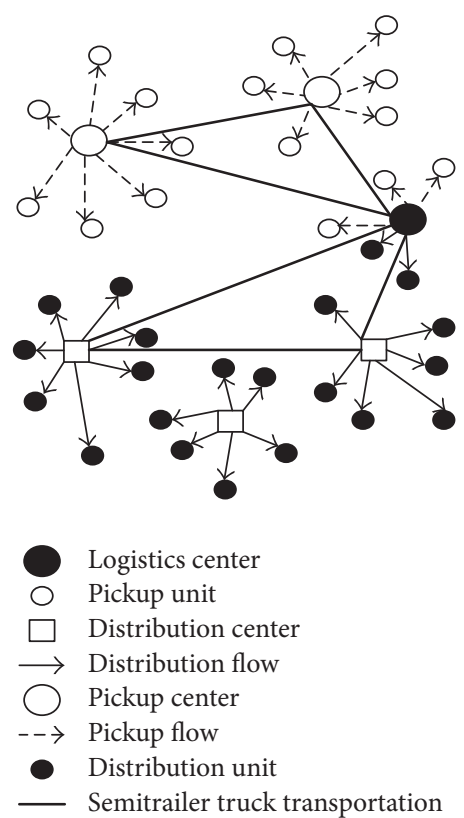

(c) After logistics network optimization

FIGURE 1: Logistics network diagram.

In summary, our research mainly contributes to the domain in the following directions: (1) a mixed integer programming model is established to interpret the two-echelon heterogeneous cooperative logistics network (THCLN) and minimize the total cost of nonempty coalitions; (2) variations in facilities transportation needs, routes, and required fleet size are considered as a result of cooperation, and a model is built to calculate payoffs based on members participation in the collective profit; (3) this paper combines the Genetic Algorithm and the Particle Swarm Optimization heuristics to propose a GA-PSO hybrid algorithm and find the most appropriated logistics facility for each customer clustering unit. (4) A practical study is adopted to support the effectiveness of the implemented approach in real world.

\section{Problem Statement and Related Definition}

2.1. Problem Description. THCLN increases the sustainability of logistics networks through the partitioning of customers, the sharing of transportation resources, and the reduction of greenhouse gas emissions. Figure 1 shows the network's structural changes which occur as logistics facilities agree to cooperate. Owing to their comprehensive functions, this article assigns the coordination of coalitions to LCs. For the synergy purposes, customer units can be redistributed among LCs, DCs, and PCs in accordance with the required type of service and their geographical locations. Therefore, distribution units can only be reassigned to LCs or DCs, whereas pickup units should be allocated to LCs or PCs. Transportation at the first echelon is assured by semitrailer trucks, and small vehicles are used to serve customers.
From a global point of view, Figure 1(a) illustrates a logistics networks characterized by the complexity of both distribution and pickup flows. In fact, companies usually invest in marketing events like advertisements and offer discounts in order to attract more customers to buy their products or require their services. Owing to these events, several customers overlook factors like distance or road conditions and make decisions which might prove irrational over a long period of time. Consequently, with the development of city, the increase in population and the number of logistics facilities, the entire network gains in complexity and gives rise to phenomena like traffic congestion and pollution. Despite the existence of cases where customers change suppliers as new facilities are established closer to them, others maintain partnerships due to loyalty entertained for years. As our proposed initial network presents a two-echelon transportation pattern, we also take into account semitrailer truck trips between PCs and DCs in order to highlight the existence of prior partnerships among centers. Those semitrailer truck transportations in Figure 1(b) can be interpreted as follows: PCs usually collect semifinished products from customers and process them before supplying DCs.

2.2. Logistics Network Optimization Architecture. Solving the THCLN consists in encouraging logistics facilities to synergize and share customers, semitrailer trucks, and vehicles. In addition, agreements should be achieved between PCs and DCs to use LCs as upper-level facility and redirect initial product flows through logistics centers. After the optimization, the network would be more organized, as shown in Figure 1(c), and long distance distributions or 


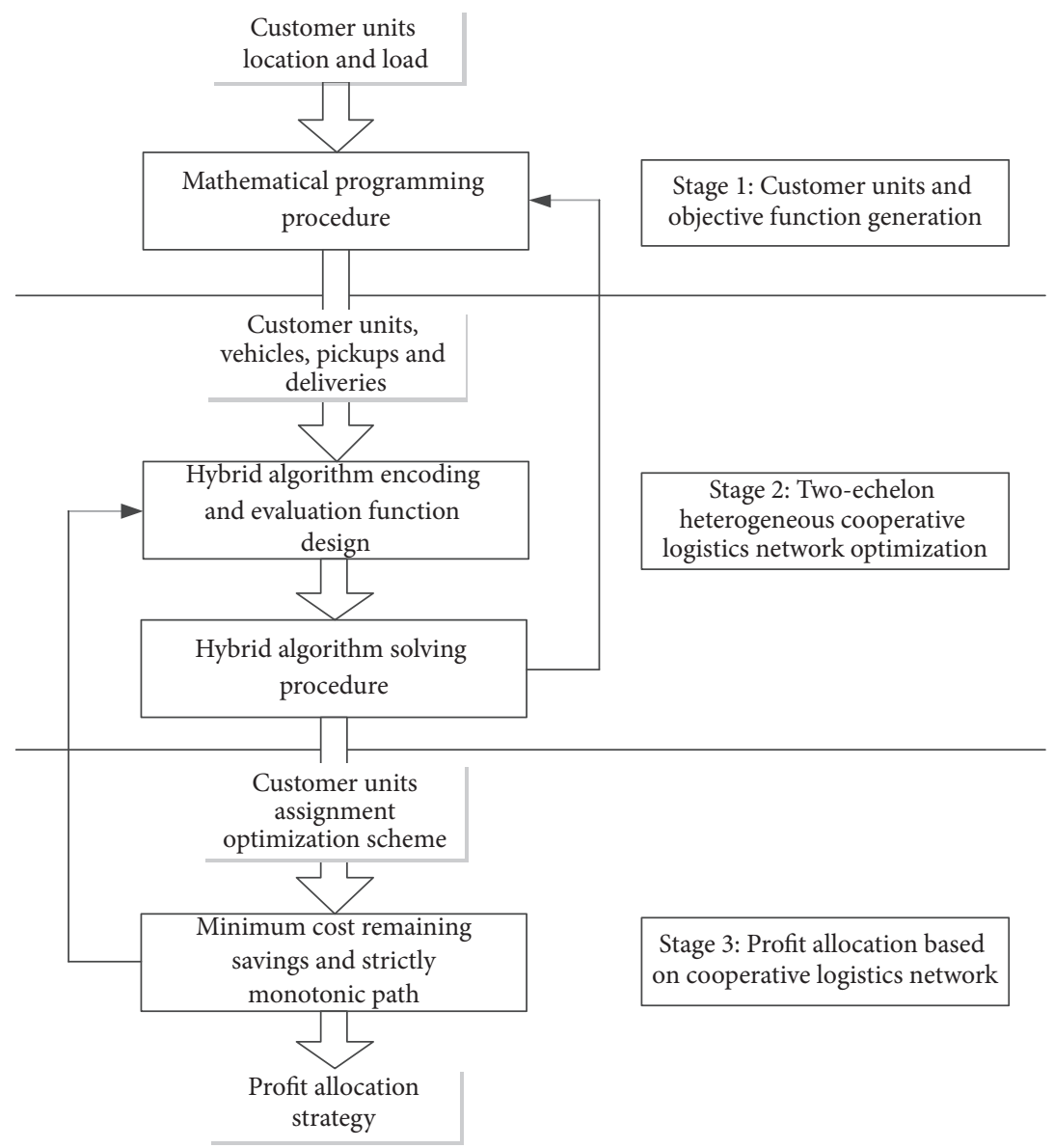

FIGURE 2: Logistics network optimization architecture.

pickups can be avoided within the same city. Products at the first echelon are still transported by means of semitrailer trucks and distribution or pickup operations carried out with a homogeneous fleet of vehicle.

The logistics network optimization architecture includes a three-phase solution approach shown in Figure 2. First, customer units' location and related demand data are collected and clustered, and a mixed integer linear mathematical model is proposed to interpret real world operations. Then, considering the available type of vehicle fleet as well as their capacities, GA and PSO are integrated to address the two-echelon heterogeneous logistics network optimization problem. Results obtained after running GA-PSO are used to compute the gap between objective function values before and after collaboration. Further, the Minimum Cost Remaining Savings method and the strictly monotonic path selection principles are consecutively applied. Their objectives are to distribute the cost savings among logistics facilities and evaluate the stability of one or multiple coalitions in the network.

2.3. Related Definition. In order to interpret the proposed problem into a mathematical model for further computations, some related variables are defined as follows:
$I\{i \mid i=1,2,3, \ldots, m\}$ denotes the set of PCs, and $m$ is the total number of PCs.

$J\{j \mid j=1,2,3, \ldots, n\}$ denotes the set of DCs, and $n$ is the total number of DCs.

$U\{u \mid u=1,2,3, \ldots, a\}$ denotes the set of pickup units in the two-echelon heterogeneous logistics network, and $a$ is the total number of pickup units.

$U^{\prime}\left\{\left|u^{\prime}\right| u^{\prime}=1,2,3, \ldots, b\right\}$ denotes the set of distribution units in the two-echelon heterogeneous logistics network, and $b$ is the total number of distribution units.

$F\{f \mid f=m+1\}$ expresses that LCs only apply their pickup capacities in the two-echelon heterogeneous logistics network.

$F^{\prime}\left\{f^{\prime} \mid f^{\prime}=n+1\right\}$ expresses that LCs only apply their delivery capacities in the two-echelon heterogeneous logistics network.

$Q_{u, i}$ expresses the pickup quantity from the pickup unit $u$ to LC or PC with in a working period, $i \in I \cup F$.

$Z_{j, u^{\prime}}$ denotes the delivery demand transported from LC or DC to the distribution unit $u^{\prime}$ within a working period, $j \in J \cup F^{\prime}$. 
$S_{u, i}$ denotes the distance from the pickup unit $u$ to LC or PC, $i \in I \cup F$.

$S_{j, u^{\prime}}^{\prime}$ denotes the distance from LC or DC to the distribution unit $u^{\prime}, j \in J \cup F^{\prime}$.

$p_{i, h}$ expresses the transport quantity from pickup facility $i$ to $h$ within a working period, $i, h \in I \cup F$, and $h \neq i$.

$d_{j, h^{\prime}}$ expresses the transport quantity from distribution facility $j$ to $h^{\prime}$ within a working period, $j, h^{\prime} \in$ $J \cup F^{\prime}$ and $h^{\prime} \neq j$.

$p_{i}$ expresses the pickup capacity of the $i$ th PC or LC within a working period, where $i \in I \cup F$.

$d_{j}$ expresses the delivery capacity of the $j$ th DC or LC within a working period, where $j \in J \cup F^{\prime}$.

$T_{p}$ denotes the total pickup capacity of LC and DCs and can be expressed as $P=\sum_{i} p_{i}$, where $i \in I \cup F$.

$T_{d}$ denotes the total delivery capacity of LC or PCs and can be expressed as $D=\sum_{j} d_{j}$, where $j \in J \cup F^{\prime}$.

$P_{u}$ expresses the pickup quantity of the pickup unit $u$ within one working period.

$D_{u^{\prime}}$ expresses the delivery demand of the distribution unit $u^{\prime}$ within one working period.

$T$ expresses the total pickup quantity from all pickup units within one working period and can be expressed as $T=\sum_{u} q_{u}$, where $u \in U$.

$T^{\prime}$ expresses the total delivery demand of all distribution units within one working period, and can be expressed as $T^{\prime}=\sum_{u^{\prime}} q_{u^{\prime}}$, where $u^{\prime} \in U$;

$R\left\{r_{i, u} \mid r_{i, u}=0,1, i \in I \cup U, U \in U\right\}$ expresses the pickup relation matrix, where $r_{i, u}=1$ indicates that LC or PC collects goods from pickup unit $u$, and $r_{i, u}=$ 0 implies that LC or PC does not collect goods from the pickup unit.

$R^{\prime}\left\{r_{j, u^{\prime}}^{\prime} \mid r_{j, u^{\prime}}^{\prime}=0,1, j \in J \cup G^{\prime}, u^{\prime} \in U^{\prime}\right\}$ expresses the distribution relation matrix, where $r_{j, u^{\prime}}^{\prime}=1$ indicates that $\mathrm{LC}$ or $\mathrm{DC}$ delivers goods to distribution unit $u^{\prime}$ and $r_{j, u^{\prime}}^{\prime}=0$ implies that LC or DC does not delivery goods to the distribution unit $u^{\prime}$.

$L_{s}$ denotes the loading capacity of a semitrailer truck.

$L_{v}$ denotes the loading capacity of a vehicle.

$f_{s r}$ denotes the freight rate of a semitrailer truck (dollar/pallet-kilometer).

$f_{v r}$ denotes the freight rate of a vehicle (dollar/ pallet-kilometer).

$M_{s}$ denotes the average annual maintenance cost of a semitrailer truck (dollar/pallet-kilometer).

$M_{v}$ denotes the average annual maintenance cost of a vehicle (dollar/pallet-kilometer).

$\gamma$ expresses the variable cost coefficient of LCs $\gamma_{i}$ expresses the variable cost coefficient of DCs.

$\gamma_{j}$ expresses the variable cost coefficient of PCs.

$\tau$ expresses the number of working periods.

$L_{\text {max }}$ expresses the maximum delivery distance.

$C$ expresses the cost savings provided by the coordinator when logistics facilities agree to cooperate.

$G$ expresses the government incentive provided to logistics facilities in case of cooperation.

$L_{i, h}$ denotes the distance from the LC or PC $i$ to $h$, $i, h \in I \cup F, i \neq h$.

$L_{j, h^{\prime}}^{\prime}$ denotes the distance from the LC or DC $j$ to $h^{\prime}$, $j, h^{\prime} \in J \cup F^{\prime}, j \neq h^{\prime}$.

$N_{t}$ denotes the number of trips of the vehicle within one working period.

$N_{t}^{\prime}$ denotes the number of trips of the semitrailer truck within one working period.

$X_{i}$ denotes the cooperation relation between PCs and the coordinator; if PC $i$ agrees to cooperate with LC, set $X_{i}=1$; otherwise set $X_{i}=0$.

$X_{j}$ denotes the cooperation relation between DCs and the coordinator; if DC agrees to cooperate with LC, set $X_{j}=1$; otherwise set $X_{j}=0$.

$\varphi$ denotes the fixed cost of LCs.

$y_{i}$ denotes the fixed cost of PC $i$.

$y_{j}$ denotes the fixed cost of DC $j$.

$N=\{0,1,2, \ldots, n\}$ denotes the sequence set of $\mathrm{LC}$ and DCs, where

$$
\left\{\begin{array}{cccccc}
0 & 1 & 2 & \cdots & n & 0 \\
\mathrm{LC} & \mathrm{DC}_{1} & \mathrm{DC}_{2} & \cdots & \mathrm{DC}_{n} & \mathrm{LC}
\end{array}\right\}
$$

with 0 representing LC. Notice that any DC can be the first node in coalitions without LC.

$q_{\beta, \alpha}$ denotes the variable transportation quantity between LCs and DCs, $\alpha, \beta \in N, \alpha<\beta$. For example,

$$
\left\{\begin{array}{cccc}
0 & 1 & 2 & 0 \\
\mathrm{LC} & \mathrm{DC}_{1} & \mathrm{DC}_{2} & \mathrm{LC}
\end{array}\right\}
$$

denotes that the semitrailer truck departs from LC, visits $\mathrm{DC}_{1}$ and $\mathrm{DC}_{2}$, and returns to $\mathrm{LC} ; q_{1,2}$ expresses the variable transport quantity from $\mathrm{DC}_{1}$ to $\mathrm{DC}_{2}$.

\section{Model Formulation}

The THCLN optimization problem is interpreted into a mixed integer linear programing model to minimize the total cost. The objective function is the aggregation of logistics facilities fixed and variable operations costs as well as transportation and maintenance costs of semitrailer trucks and vehicles. $W_{1}$ is the total transportation and maintenance 
cost among LCs and PCs (if cooperation occurs) within a working period; $W_{2}$ is the total transportation and maintenance cost among LCs and DCs (if cooperation occurs) within a working period; $W_{3}$ is the total transportation and maintenance cost of vehicles from LC or PC to each pickup unit and LC or DC to each distribution unit within a working period; and $W_{4}$ is the result of subtracting cooperation discounts from the sum of fixed and variable costs within a working period. The objective function can be expressed as follows:

$$
\min W=W_{1}+W_{2}+W_{3}+W_{4}
$$

with

$$
\begin{aligned}
& W_{1}=\sum_{i, h \in I \cup F, i \neq h}\left(p_{i, h} \times f_{s v} \times L_{i, h} \times 2 \times N_{t}^{\prime}+\frac{p_{i, h}}{L_{s}}\right. \\
& \left.\times \frac{M_{s}}{T}\right) \\
& W_{2}=\sum_{j, h^{\prime} \in J \cup F^{\prime}, j \neq h^{\prime}} d_{j, h^{\prime}} \times f_{s r} \times L_{j, h^{\prime}}^{\prime} \times N_{t}^{\prime}+\sum_{\alpha, \beta \in J} q_{\beta, \alpha} \\
& \times f_{s r} \times L_{j, h^{\prime}}^{\prime} \times N_{t}^{\prime} \\
& +\frac{\sum_{j, h^{\prime} \in J \cup F^{\prime}, j \neq h^{\prime}} d_{j, h^{\prime}}+\sum_{\alpha, \beta \in J} q_{\beta, \alpha}}{L_{s}} \times \frac{M_{s}}{T} \\
& W_{3}=\sum_{u \in U} \sum_{i \in I \cup F}\left(Q_{u, i} \times r_{i, j} \times S_{u, i} \times 2 \times f_{v r} \times N_{t}\right) \\
& +\frac{\sum_{i \in I \cup F, u \in U}\left(Q_{u, i} \times r_{i, u}\right)}{L_{v}} \times \frac{M_{v}}{T} \\
& +\sum_{u^{\prime} \in U^{\prime}} \sum_{j \in J \cup F^{\prime}}\left(Z_{j, u^{\prime} \times} r_{j, u^{\prime}}^{\prime} \times S_{j, u^{\prime}}^{\prime} \times 2 \times f_{v r} \times N_{t}\right) \\
& +\frac{\sum_{j \in J \cup F^{\prime}, u^{\prime} \in U^{\prime}}\left(Z_{j, u^{\prime}} \times r_{j, u^{\prime}}^{\prime}\right)}{L_{v}} \times \frac{M_{v}}{T} \\
& W_{4}=\sum_{i \in I} y_{i}+\sum_{j \in J} y_{j}+\varphi+\sum_{i \in I} \sum_{u \in U}\left(Q_{u, i} \times r_{i, u} \times \gamma_{i}\right) \\
& +\sum_{j \in J} \sum_{u^{\prime} \in U^{\prime}}\left(Z_{j, u^{\prime}} \times r_{j, u^{\prime}}^{\prime} \times \gamma_{j}\right)+\left[\sum_{i \in F} \sum_{u \in U}\left(Q_{u, i} \times r_{i, u}\right)\right. \\
& \left.+\sum_{j \in F^{\prime}} \sum_{u^{\prime} \in U^{\prime}}\left(Z_{j, u^{\prime}} \times r_{j, u^{\prime}}^{\prime} \times \gamma_{j}\right)\right] \times \gamma-\left(\sum_{i \in I \cup F} X_{i}\right. \\
& \left.+\sum_{j \in J \cup F^{\prime}} X_{j}\right)(C+G)
\end{aligned}
$$

subject to

$$
\begin{aligned}
\sum_{i \in I \cup G} r_{i, u} & =1, \quad \forall u \in U \\
\sum_{j \in J \cup G^{\prime}} r_{j, u^{\prime}} & =1, \quad \forall u^{\prime} \in U^{\prime} \\
r_{i, u} & =\{0,1\}, \\
r_{j, u^{\prime}} & =\{0,1\}
\end{aligned}
$$

$\forall i \in I \cup F, j \in J \cup F^{\prime}, u \in U, u^{\prime} \in U^{\prime}$

$$
\begin{aligned}
p_{i} & =\sum_{u \in U} r_{i, u} \times Q_{u, i}, \quad \forall i \in I \cup F \\
d_{j} & =\sum_{u^{\prime} \in U^{\prime}}\left(r_{j, u^{\prime}}^{\prime} \times Z_{j, u^{\prime}}\right), \quad \forall j \in J \cup F \\
P & =\sum_{i \in I \cup F} r_{i, u} \times Q_{u, i}, \quad \forall u \in U \\
D & =\sum_{j \in J \cup F^{\prime}}\left(r_{j, u^{\prime}}^{\prime} \times Z_{j, u^{\prime}}\right), \quad \forall u^{\prime} \in U^{\prime} \\
S_{u, i}>L_{\max }, & \\
r_{i, u} & =0, \\
Q_{u, i} & =0
\end{aligned}
$$

$$
\begin{aligned}
& S_{j, u^{\prime}}^{\prime}>L_{\max }, \\
& r_{j, u^{\prime}}^{\prime}=0, \\
& Z_{j, u^{\prime}}=0,
\end{aligned}
$$

$$
\begin{aligned}
& \quad j \in J \cup F^{\prime}, \forall u^{\prime} \in U^{\prime} \\
& Q_{u, i}, Z_{j, u^{\prime}} \geq 0, \\
& \forall i \in I \cup F, \forall j \in J \cup F^{\prime}, u \in U, u^{\prime} \in U^{\prime} .
\end{aligned}
$$

The objective function value is calculated using (3). Equations (4) are the subpart of the cost function and represent transportation, maintenance, and fixed and variable costs. Constraints (5)-(6) ensure that a customer unit is only served by a center. Constraint (7) introduces the binary relation variable between DCs, PCs, and their corresponding customers. Constraints (8)-(9) guarantee that delivery and pickup centers, respectively, meet their customers' needs. Constraint (10) stipulates that pickup activities can either be performed by LCs or PCs, whereas constraint (11) allows only LCs and DCs to deliver products to customer units. Constraints (12)-(13) regulate the maximum travelling distance of pickup and delivery vehicles. Constraint (14) defines nonnegative variables. 
TABLE 1: Two-dimensional chromosome encoding table.

\begin{tabular}{|c|c|c|c|c|c|c|c|c|c|c|c|}
\hline Pickup/distribution unit & 1 & 2 & $\cdots$ & $i$ & $\cdots$ & $L^{\prime}$ & $L^{\prime}+1$ & $\cdots$ & $j$ & $\cdots$ & $L$ \\
\hline Logistics facility number & $f_{k, 1}$ & $f_{k, 2}$ & $\cdots$ & $f_{k, i}$ & $\ldots$ & $f_{k, L^{\prime}}$ & $f_{k, L^{\prime}+1}$ & $\cdots$ & $f_{k, j}$ & $\ldots$ & $f_{k, L}$ \\
\hline
\end{tabular}

\section{The Hybrid Algorithm Solving Procedure}

The Genetic Algorithm (GA) and Particle Swarm Optimization (PSO) algorithm are combined to design a GA-PSO hybrid algorithm used to address the two-echelon heterogeneous logistics network optimization model. GA is an evolutionary computing approach used to mimic the natural selection procedure and study combinatorial optimization problems $[17,23]$. PSO is one of the swarm intelligence stochastic evolutionary metaheuristic approaches, proposed by Kennedy and Eberhart [24]. PSO is inspired by observations of the social behavior of bird flocking, fish schooling, and so on, based on swarm theory, and can be used to optimize the local and global locations according to the particle's position and velocity. Traditionally, these two algorithms have the merits of being population-based heuristics with the ability to evaluate the fitness of each possible solution. Through local and global search capabilities, GA and PSO have been successfully applied to solve problems in management, engineering, and pure science domains. Considering their individual abilities, we propose GA combined with PSO in a hybrid algorithm, which inherits the merits of both approaches and increases the probability of obtaining optimal solutions.

As a hybrid algorithm, GA-PSO is applied to meet the requirements of logistics network optimization for more complex algorithms. Existing traditional heuristics have already been proved efficient, but the increasing complexity of logistics networks limits their capacity at finding nearoptimal solutions. In consequence, properly integrating different methods into a hybrid solution approach like GAPSO can effectively improve the quality of optimization results. Other hybrid heuristics exist but some of them display weaknesses compared to GA-PSO in terms of performance. For example, Chen et al. [25] embedded a local search heuristic in the Ant Colony Optimization (ACO) algorithm to improve the solution search ability of ACO and address a task assignment problem. The proposed methodology had a satisfying global search capability but the local search presented insufficiencies. Combining GA and PSO on the contrary ensures good performances at both local and optimum search level. This integration can improve the optimization procedure and reduce the number of necessary iterations. The relevant operations and processes of the proposed hybrid algorithm are illustrated in sections below.

4.1. Hybrid Algorithm Encoding Scheme and Evaluation Function Design. Hybrid algorithm encoding and objective function designing are the key issues in the optimization operation processes [26]. In the two-echelon heterogeneous logistics distribution network, the pickup/delivery capacity of each logistics facility and the transportation cost among logistics facilities should be taken into account. Therefore, a two-dimensional chromosome encoding is proposed in this study. The first dimension of the chromosomes is denoted as a sequence of natural numbers: $1,2,3, \ldots, i, \ldots, L^{\prime}, L^{\prime}+$ $1, \ldots, j, \ldots, L$, and $L^{\prime}$ is the total number of pickup units, and $\left(L-L^{\prime}\right)$ is the total number of distribution units. The second dimension is the chromosome (particle) position. The chromosome position can be defined as the sequence number of logistics facilities including LC, DC, and PC that are assigned to serve each corresponding pickup unit and distribution unit. $f_{k, i}$ indicates the logistics facility including logistics center or pickup center assigned to the ith pickup unit in the $k$ th chromosome, while $f_{k, j}$ represents the logistics facility including logistics center or distribution center assigned to the $j$ th distribution unit in the $k$ th chromosome. The two-dimensional chromosome encoding table is shown in Table 1. For example, if we set $f_{k, i}=0,1,2, f_{k, j}=0,3,4,5$, and $i=1,2,3, \ldots, L^{\prime}, j=L^{\prime}+1, \ldots, L, f_{2,3}=2$ denotes that the PC 2 is assigned to the third pickup unit in the second chromosome, if $L^{\prime}=30$, then $f_{2,35}=3$ indicates that the DC 3 is assigned to the 35 th distribution unit in the second chromosome.

The chromosome encoding method shown in Table 1 makes sure that each pickup/distribution unit can be served by a certain logistics facility, in other words, the pickup/distribution service can be correspondingly completed by LC, PC or DC. In addition, using the chromosome encoding method to initialize populations, the computational complexity can effectively be simplified. For example, assume there are four pickup units, five distribution units, one LC, two PCs, and three DCs in the two-echelon heterogeneous logistics distribution network. The first chromosome can be generated randomly as follows: 012035405 , where pickup units 1, 4 and distribution unit 8 are served by LC, pickup units 2 and 3 are served by PC 1 and PC 2, respectively, and distribution units 5 and 7 are served by DC 3 and DC 5, respectively. The initial fitness function value can be computed based on the objective function. However, some randomly generated chromosomes in the initial population may not satisfy requirements like maximum delivery distance limit, missing the PCs or DCs, and so on. Therefore, new chromosomes need to be generated in order to substitute the previous unqualified chromosomes. To evaluate the effectiveness of our hybrid algorithm, the fitness function needs to be properly defined, and the unqualified chromosomes should be penalized. Set the unqualified chromosome number as $\gamma_{k}=\gamma_{k}+1$, with the initial value of $\gamma_{k}$ being equal to 0 . The objective function of the $k$ th chromosome is $T C_{k}$, and the fitness function can be formulated as follows:

$$
F_{k}=\frac{1}{T C_{k}+\gamma_{k} \times M},
$$


where $M$ is the penalty weight for each unqualified chromosome (particle), and it can be defined relatively to the value of the objective function.

\subsection{GA Updates and the Corresponding PSO Operations.} GA updates mechanism including the corresponding PSO operations is presented in the hybrid algorithm procedure. The fitness function value and a cumulative probability are firstly calculated for the Genetic Algorithm. Afterwards, the selection, crossover, mutation, and PSO operations are conducted sequentially before the offspring is released into the new population. In the selection process, the roulette wheel selection is proposed based on the elevated chromosomes fitness function value. In the crossover process, a random vector including 0 and 1 is presented to choose the genes corresponding to 0 from the primary parent and the ones corresponding to 1 from the second parent; the offspring can be created by combinations [27]. For example, consider a network where there are four pickup units, five distribution units, one LC, two PCs, and three DCs, the

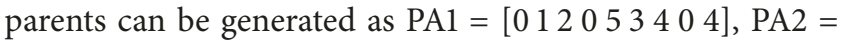

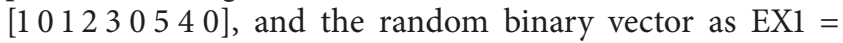

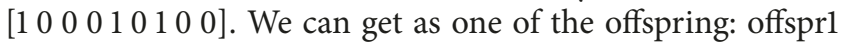
$=\left[\begin{array}{llllllll}1 & 1 & 2 & 0 & 3 & 3 & 5 & 0\end{array}\right]$. Nevertheless, the offspring chromosomes may not satisfy all the constraints. Therefore, the binary vectors need to be regenerated until the new chromosomes are qualified. The process of mutation occurs after the crossover: two particular orders of genes are reversed for pickups and deliveries in a chromosome. The inversed chromosomes including genes only reserved for pickups, those only reserved for deliveries, and the ones reserved for both pickups and deliveries are compared with the original chromosome, and the optimal one is selected using the mutation operation.

The PSO operations update both location and velocity information in our proposed algorithm. The position of each particle (chromosome) is presented as $x_{k}^{t}$ where $k=$ $1,2, \ldots, N(N$ is population size) and $t$ is the iteration number. The corresponding velocity of particle is $v_{k}^{t}$. The position is evaluated in each iteration, and if the fitness function value is better than that of previous iterations, the position is updated as the "pbest". Meanwhile, the global best position of current iteration is updated as "gbest". The initial individual best position "pbest" and the global best position "gbest" are determined based on the initial population after GA operations. The related formulas to update both the velocity and position are presented as follows:

$$
\begin{aligned}
v_{k}^{t+1} & = \begin{cases}w \times v_{k}^{t}+c_{1} \times \operatorname{rand}(t) \times\left(\text { pbest }_{k}^{t}-x_{k}^{t}\right)+c_{2} \times \operatorname{rand}(t) \times\left(\text { gbest }_{k}^{t}-x_{k}^{t}\right) & -V_{p} \leq v_{k}^{t+1} \leq V_{p} \\
-V_{p}+2 \times V_{p} \times \operatorname{rand}(t) & \text { others }\end{cases} \\
x_{k}^{t+1} & = \begin{cases}\operatorname{fix}\left(x_{k}^{t}+v_{k}^{t+1}\right) & -V_{p} \leq x_{k}^{t+1} \leq V_{p} \\
\operatorname{rand} \operatorname{int}\left[0, T_{p}^{\prime}\right] & \text { others }\end{cases} \\
v_{k}^{t+1} & = \begin{cases}w \times v_{k}^{t}+c_{1} \times \operatorname{rand}(t) \times\left(\text { pbest }_{k}^{t}-x_{k}^{t}\right)+c_{2} \times \operatorname{rand}(t) \times\left(\text { gbest }_{k}^{t}-x_{k}^{t}\right) & -V_{d} \leq v_{k}^{t+1} \leq V_{d} \\
-V_{d}+2 \times V_{d} \times \operatorname{rand}(t) & \text { others }\end{cases} \\
x_{k}^{t+1} & = \begin{cases}\operatorname{fix}\left(x_{k}^{t}+v_{k}^{t+1}\right) & -V_{d} \leq x_{k}^{t+1} \leq V_{d} \\
\operatorname{rand} \operatorname{int}\left[0, T_{d}^{\prime}\right] & \text { others, }\end{cases}
\end{aligned}
$$

where formulas (16) and (17) are used to update both the velocity and position for pickup services and formulas (18) and (19) are used to update both the velocity and position for delivery services. $c_{1}$ and $c_{2}$ are two acceleration coefficients. $\operatorname{rand}(\cdot)$ represents a random fraction between 0 and 1 . pbest $t_{k}^{t}$ denotes the individual best position of particle $k$ at the $t$ th iteration, and gbest ${ }_{k}^{t}$ is the global best position of particle $k$ at the $t$ th iteration. fix $(\cdot)$ assures that the position of each particle is an integer. $V_{p}$ and $V_{d}$ represent allowable maximum velocity for pickup and delivery services, respectively. rand int $\left[0, T_{p}^{\prime}\right]$ denotes an integer between 0 and $T_{p}^{\prime}$, and rand int $\left[0, T_{d}^{\prime}\right]$ denotes an integer between 0 and $T_{d}^{\prime}$, where $T_{p}^{\prime}$ and $T_{d}^{\prime}$ represent the numbers of logistics facility set for pickup service and delivery service, respectively. $w$ is the inertia weight and can be formulated as follows:

$$
w=\frac{\left(w_{\text {int }}-w_{\text {end }}\right) \times\left(S_{\max }-t\right)}{S_{\max }}+w_{\text {end }},
$$

where $S_{\max }$ indicates the maximum number of iterations, $w_{\text {int }}$ denotes the initial inertia weight, and $w_{\text {end }}$ denotes the inertia weight for the maximum number of iterations.

4.3. Hybrid Algorithm Procedure. Based on the features described above, the hybrid algorithm procedure is described in Figure 3 and the main steps can be detailed as follows.

Step 1 (algorithm initialization). In the hybrid algorithm procedure, 0 denotes that the logistics center, which can be used for pickup service and delivery service. An integer within $\left[0, T_{p}^{\prime}\right]$ or $\left[0, T_{d}^{\prime}\right]$ is randomly chosen and assigned to the gene position in each chromosome, and check whether 


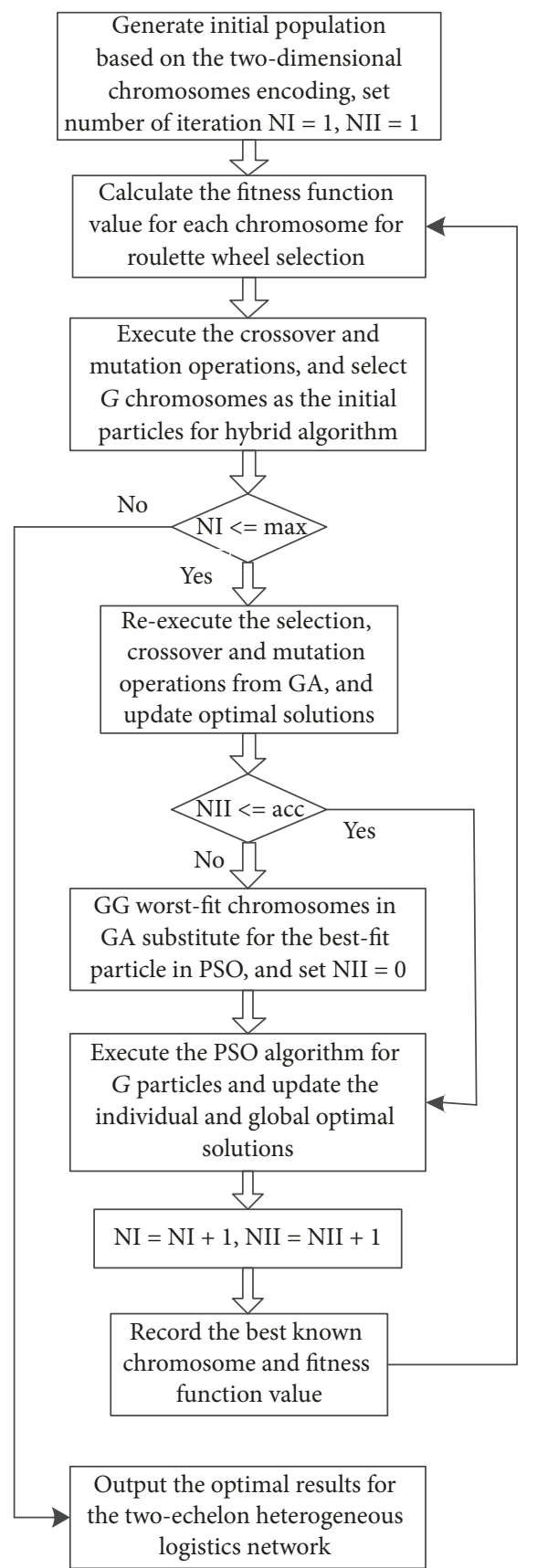

FIGURE 3: The flowchart of hybrid algorithm procedure.

the chromosomes fulfill the requirement or not. In case the condition is not respected, new chromosomes are generated until requirements are fulfilled, and calculate the fitness function value $F_{k}$ based on formula (15).

Step 2. Execute the roulette wheel selection based on the elevated chromosomes fitness function value, execute crossover and mutation operations based on the crossover probability pc and the mutation probability pm, respectively, and update the optimal solutions and new chromosomes.

Step 3. Select the $G$ chromosomes as the initial particles in the population, conduct the PSO operations as part of the hybrid algorithm, and calculate the individual optimal solution pbest $_{k}^{t}$ and the global optimal solution gbest $t_{k}^{t}$.

Step 4. Repeat the following steps until the maximum number of iterations is reached or the termination conditions are satisfied.

Step 4.1. Reexecute the selection, crossover, and mutation operations from GA; if the current fitness function value from the new offspring chromosomes is better than with the parents, update the optimal solutions and new chromosomes.

Step 4.2. Determine whether the number of iterations has achieved the periodic acc or not; if yes, replace the total $G G$ of worst-fit chromosomes from GA with the corresponding number of best-fit particles from PSO algorithm; continue PSO with $G$ particles, and update pbest ${ }_{k}^{t}$ and gbest ${ }_{k}^{t}$; otherwise, the exchange procedure will not be conducted; execute the PSO algorithm on $G$ particles and update the individual and global optimal solutions.

Step 5. If the number of iterations achieves the maximum number of iterations, the loop procedure will be finished. The current best known particle (chromosome) and fitness function value will be chosen as the optimal solution; otherwise, return to Step 2 to continue.

Step 6. Calculate and select the optimal solution from all feasible chromosomes. The optimal solution will be the final result for the two-echelon heterogeneous logistics network.

In the above hybrid algorithm procedure, the GA operations and PSO operations are reasonably combined by exchanging worst-fit solutions and best-fit solutions between GA and PSO algorithms. Therefore, it enhances the optimization capability of the hybrid algorithm and provides a more robust local and global search capability.

\section{Profit Allocation Strategy}

5.1. Minimum Cost Remaining Savings Model. The MCRS model is a game theoretic method used to solve cost or profit allocation problems [28]. The selection of MCRS to calculate logistics facilities profits is due to its well-known merits summarized as follows: (1) the model is simple to compute and each to implement; (2) results are generally fairly balanced and presented in a single vector, easy to visualize; (3) MCRS guarantees both individual and collective profit rationality.

The method proceeds to a two-step distribution, where a portion of the collective profit is distributed before the allocation of the remaining part in order to measure members' contributions. The upper and lower bound vectors $A_{\min }=$ $\left\{A_{1 \min }, \ldots, A_{j \min }, \ldots, A_{n \min }\right\}$ and $A_{\max }=\left\{A_{1 \max }, \ldots\right.$, $\left.A_{j \max }, \ldots, A_{n \max }\right\}$ of the allocation scheme are first determined according to highest and lowest possible profit of each participant. $A_{j \max }$ denotes the maximum profit of logistics facility $j$ and $A_{j \min }$ denotes its minimum value. Assume that $A_{j}^{*}$ represents the actual profit of logistics facility $j, v(s)$ being 
the collective profit of alliance $S$ and $v(z)$ the total benefit of the grand coalition. The value of the facility $j$ 's profit is determined by the following:

$$
\begin{aligned}
A_{j}^{*}= & A_{j \min }+\frac{\left(A_{j \max }-A_{j \min }\right)}{\sum_{j \in N}\left(A_{j \max }-A_{j \min }\right)} \\
& \times\left(V(N)-\sum_{j \in N} A_{\min }\right),
\end{aligned}
$$

where

$$
\begin{aligned}
\lambda & =\frac{\left(A_{j \max }-A_{j \min }\right)}{\sum_{j=1}\left(A_{j \max }-A_{j \min }\right)} \\
\sum_{i=1}^{n} A_{i} & =V(N),
\end{aligned}
$$

where $V(N)$ refers to the hyperplane and $\lambda$ is a coefficient used to balance the results. The bound vector's variables $A_{\text {jmax }}$ and $A_{j \min }$ can be solved by the following linear programming model:

$$
\begin{array}{ll}
\max & A_{j}, \\
\min & A_{j} \\
\text { s.t. } & \sum_{j \in s} A_{j} \geq v(s) \\
& \sum_{j \in z} A_{j}=v(z) .
\end{array}
$$

5.2. Strictly Monotonic Path Principles. The determination of good coalitions is subject to the verification of strictly monotonic path (SMP) principles. The multitude of coalition possibilities generally provides a wide range of profit allocation schemes. A sequence can be adopted only if the cost reduction percentage of each participant increases whenever a new facility joins. If $\pi$ is a sequence of the coalition, $\pi(i)$ can represent the position of facility $i$ in sequence $\pi . \eta(i, \pi, u)$ represents the cost reduction rate when facility $i$ joins the coalition as the $\mu$ th member and can be calculated as follows:

$$
\eta(i, \pi, u)=\frac{\phi_{i}\left(\bigcup_{\pi(\mu) \leq u, u \in s} \mu, v\right)}{C_{0} i} .
$$

The process of selecting the SMP sequence is as follows.

Step 1. Select all sequence coalitions where the cost reduction percentage conforms to the principles of SMP, that is, monotonically increasing, and find the diagonal values in the matrix.

Step 2. Find the minimum value of the diagonal line and move to the next step or choose the second smallest value if the percentages are equal. Continue until all members are searched.
Step 3. The coalition sequence yielded from Step 2 is a candidate for the best profit distribution scheme. The process should be repeated for every sequence to ensure that all the possibilities have been evaluated.

\section{Empirical Analyses}

6.1. Data Description. We conduct a practical experimentation of the proposed network optimization methodology in Chongqing city, China, to evaluate the effects of collaboration and the applicability of our approach in real situations. Real locations of one LC, two PCs, three DCs, 50 pickup units, and 70 distribution units have been selected to illustrate irrational customer allocations observed in the urban logistics network. In the cooperative network, LC plays the roles of participant and coordinator. In order to clearly define the operations of the logistics center, we represent its pickup function by $\mathrm{LC}_{\mathrm{P}}$ and the distribution function by $\mathrm{LC}_{\mathrm{D}}$. Figure 4 shows the logistics network before optimization. In order to facilitate the calculation, each distribution and pickup unit's demand is converted to the standard roll pallet quantity. The characteristics of all the logistics facilities are summarized in Table 2.

6.2. Improved ASO Parameter Setting and Optimization Results. In this section, we introduce the different parameters initialized for optimization purposes. They are separated in two categories such as objective function calculation parameters and algorithmic parameters and displayed as follows:

(1) Objective function parameters: $L_{S}=2000, L_{V}=200$, $f_{s r}=0.000625, f_{v r}=0.001875, M_{S}=5000, M_{v}=$ $1700, T=52, L=50, N_{t}=10, N t^{\prime}=5, \xi_{p 0}=1500$, $\xi_{p 1}=1800, \xi_{p 2}=1050, \xi_{d 0}=1200, \xi_{d 1}=1200, \xi_{d 2}=$ $600, \xi_{d 3}=900$. To encourage logistics facilities to cooperate, the local government provides the following as incentive: $G_{\mathrm{PC}}=1089, G_{\mathrm{PC} 1}=1144, G_{\mathrm{PC} 2}=$ $923, G_{\mathrm{DC}}=996, G_{\mathrm{DC} 1}=744, G_{\mathrm{DC} 2}=526, G_{\mathrm{DC} 3}=$ 531. The total cost savings provided if all the facilities form the grand alliance is $C=6465$.

(2) Algorithm parameters: $N=100$ is the popular size used to increase the diversity of initial chromosomes; $V_{p}=2$ expresses the maximum velocity for pickup service; $V_{d}=3$ expresses the maximum velocity for delivery service; $p_{c}=0.8$ and $p_{m}=0.02$ indicate the crossover and mutation probability, respectively; $G=$ 50 indicates chromosomes as the initial particles in the population; $G G=20$ expresses the number of chromosomes for exchanges between GA and PSO; $a c c=50$ denotes the number of iterations used for replacement between GA and PSO; $T_{p}^{\prime}=2$ and $T_{d}^{\prime}=3$ represent the numbers of logistics facility for pickup and delivery, respectively; $M=100000$ is the penalty weight for each unqualified chromosome or particle; $S_{\max }=1000$ is the maximum number of iterations; $\omega_{\text {int }}=0.8$ is the initial inertia weight and $\omega_{\text {end }}=$ 0.3 is the inertia weight of the maximum evolution generation; $c_{1}=c_{2}=2$ are coefficients used for PSO speed calculation. 
TABLE 2: Characteristics of six logistics facilities.

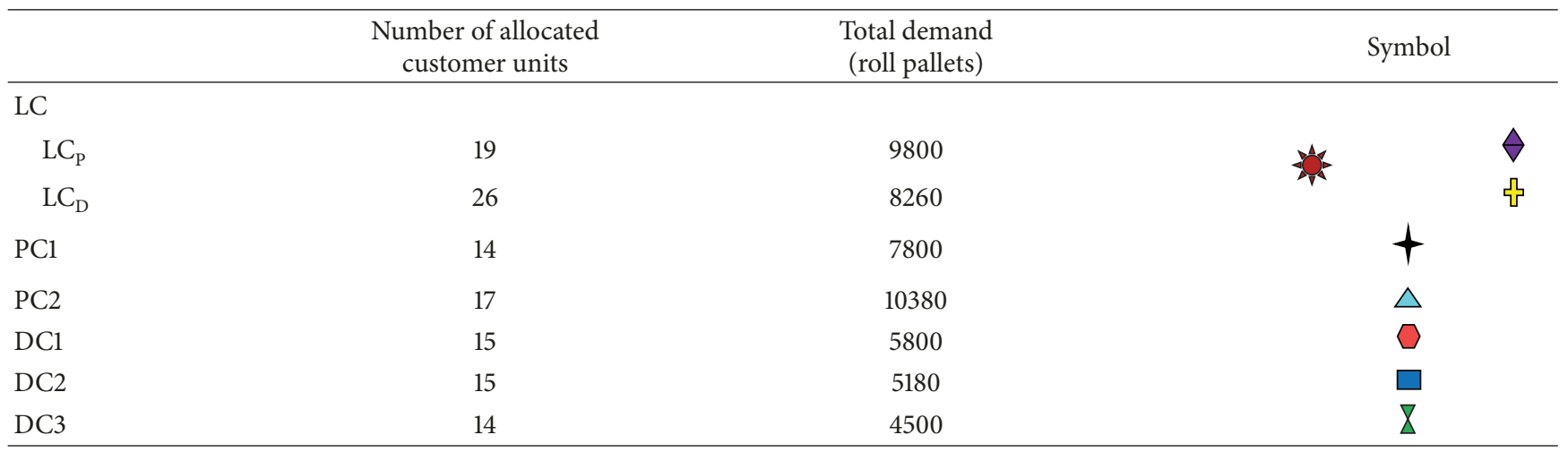

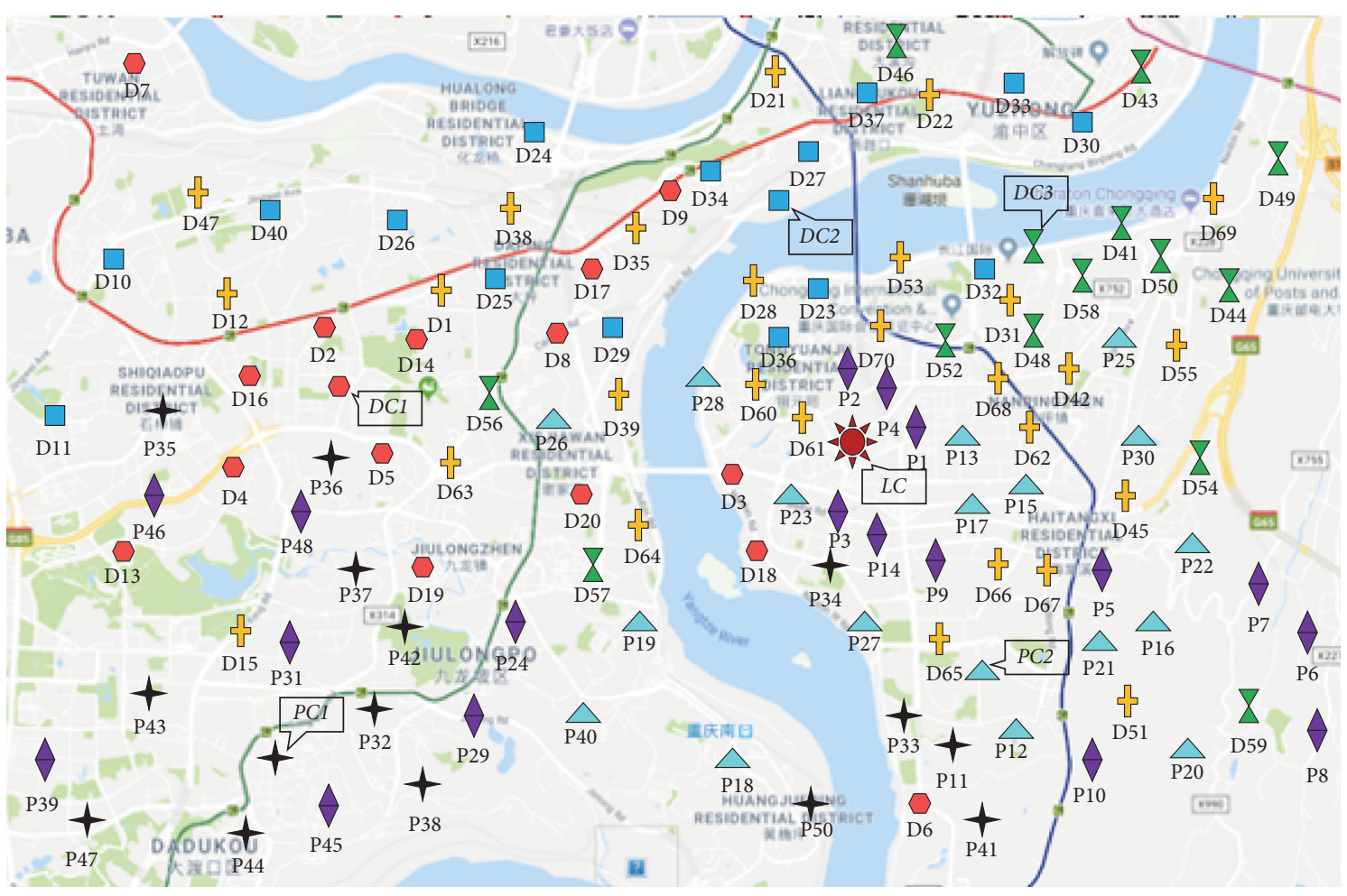

FIGURE 4: Pickup and distribution centers and units distribution diagram.

In this study, a working period consists of five working days. GA-PSO algorithm is used to assign customer units to corresponding logistics facilities and to compute the total cost in one working period. Cost savings are redistributed to participants of every coalition by applying the MCRS model. Optimization results are listed in Table 3, the initial customer units' assignment results are displayed in Table 4 , and the best customer allocation in the grand alliance is summarized in the Table 5.

Compared with the initial customer allocation presented in Table 4, the cooperative network illustrated in Table 5 shows that the number of assigned customers varies from a logistics facility to another. While some centers have observed an increase, others have been allocated less units. For example, DC2's customers units decreased from 15 to 9 , whereas PCl's customer units increased from 14 to 22. As a consequence, the total distance travelled by each facility's vehicles will decrease and savings will increase. Besides, the sharing of customers units allows LC, DCs, and PCs to reduce the number of unreasonable delivery and pickup trips, as well as the complexity of their corresponding transportation network.

For comparison purposes, we implement and test the proposed GA-PSO hybrid algorithm, CPTS (Cooperative Parallel Tabu Search), HGA (Hybrid Genetic Algorithm), and TLBO (Teaching-Learning-Based Optimization) with same data. CPTS utilizes the information interchange to accomplish the cooperation and boost both intensification and strategic diversification (James et al. 2009). HGA incorporates the elitism genetic operator and some improvement 
TABLE 3: Comparison between initial and optimized network over one working period.

\begin{tabular}{|c|c|c|c|c|c|}
\hline \multirow{2}{*}{$S$} & \multicolumn{2}{|c|}{ Initial } & \multicolumn{2}{|c|}{ Optimized } & \multirow{2}{*}{$V(t)$} \\
\hline & Cost & Demand & Cost & Demand & \\
\hline $\mathrm{LC}_{\mathrm{P}}$ & 5446 & 9800 & 4015 & 6820 & 648 \\
\hline $\mathrm{LC}_{\mathrm{D}}$ & 4980 & 10380 & 3558 & 8520 & 1422 \\
\hline $\mathrm{PC} 1$ & 5721 & 8260 & 3976 & 12000 & 1158 \\
\hline PC2 & 4613 & 7800 & 3338 & 7040 & 688 \\
\hline DC1 & 3320 & 5800 & 2081 & 9900 & 1239 \\
\hline DC2 & 2629 & 5180 & 1861 & 4860 & 769 \\
\hline DC3 & 2655 & 4500 & 1895 & 4580 & 760 \\
\hline $\mathrm{LC}_{\mathrm{P}}, \mathrm{PCl}$ & 11166 & 18060 & 8813 & 18060 & 2353 \\
\hline $\mathrm{LC}_{\mathrm{P}}, \mathrm{PC} 2$ & 10058 & 17600 & 7262 & 17600 & 2796 \\
\hline $\mathrm{LC}_{\mathrm{D}}, \mathrm{DC} 1$ & 8300 & 16180 & 5250 & 16180 & 3050 \\
\hline $\mathrm{LC}_{\mathrm{D}}, \mathrm{DC} 2$ & 7609 & 15560 & 4786 & 15560 & 2824 \\
\hline $\mathrm{LC}_{\mathrm{D}}, \mathrm{DC} 3$ & 7635 & 14880 & 4750 & 14880 & 2886 \\
\hline $\mathrm{LC}_{\mathrm{P}}, \mathrm{PC} 1, \mathrm{PC} 2$ & 15779 & 25860 & 9412 & 25860 & 4411 \\
\hline $\mathrm{LC}_{\mathrm{D}}, \mathrm{DC} 1, \mathrm{DC} 2$ & 10929 & 16180 & 7621 & 16180 & 3308 \\
\hline $\mathrm{LC}_{\mathrm{D}}, \mathrm{DC} 1, \mathrm{DC} 3$ & 10955 & 15560 & 6054 & 15560 & 4901 \\
\hline $\mathrm{LC}_{\mathrm{D}}, \mathrm{DC} 2, \mathrm{DC} 3$ & 10265 & 14880 & 4955 & 14880 & 5310 \\
\hline $\mathrm{LC}_{\mathrm{P}}, \mathrm{PC} 1, \mathrm{PC} 2, \mathrm{LC}_{\mathrm{D}}, \mathrm{DC} 1$ & 24079 & 16180 & 16434 & 16180 & 7644 \\
\hline $\mathrm{LC}_{\mathrm{P}}, \mathrm{PC} 1, \mathrm{PC} 2, \mathrm{LC}_{\mathrm{D}}, \mathrm{DC} 2$ & 23388 & 15560 & 15984 & 15560 & 7404 \\
\hline $\mathrm{LC}_{\mathrm{P}}, \mathrm{PC} 1, \mathrm{PC} 2, \mathrm{LC}_{\mathrm{D}}, \mathrm{DC} 3$ & 23414 & 14880 & 15945 & 14880 & 7470 \\
\hline $\mathrm{LC}_{\mathrm{P}}, \mathrm{PC} 1, \mathrm{PC} 2, \mathrm{LC}_{\mathrm{D}}, \mathrm{DC} 1, \mathrm{DC} 2$ & 26708 & 21360 & 18890 & 21360 & 7819 \\
\hline $\mathrm{LC}_{\mathrm{P}}, \mathrm{PC} 1, \mathrm{PC} 2, \mathrm{LC}_{\mathrm{D}}, \mathrm{DC} 1, \mathrm{DC} 3$ & 26734 & 20680 & 17225 & 20680 & 9509 \\
\hline $\mathrm{LC}_{\mathrm{P}}, \mathrm{PC} 1, \mathrm{PC} 2, \mathrm{LC}_{\mathrm{D}}, \mathrm{DC} 2, \mathrm{DC} 3$ & 26044 & 20060 & 16163 & 20060 & 9881 \\
\hline $\mathrm{LC}_{\mathrm{P}}, \mathrm{PC} 1, \mathrm{PC} 2, \mathrm{LC}_{\mathrm{D}}, \mathrm{DC} 1, \mathrm{DC} 2, \mathrm{DC} 3$ & 29363 & 25860 & 16971 & 25860 & 12392 \\
\hline
\end{tabular}

TABLE 4: Initial customer units' assignment.

\begin{tabular}{|c|c|}
\hline & Customer units allocation \\
\hline \multicolumn{2}{|l|}{$\mathrm{LC}$} \\
\hline $\mathrm{LC}_{\mathrm{P}}$ & P1 P2 P3 P4 P5 P6 P7 P8 P9 P10 P14 P24 P29 P31 P39 P45 P46 P48 P49 \\
\hline $\mathrm{LC}_{\mathrm{D}}$ & $\begin{array}{l}\text { D1 D12 D15 D21 D22 D28 D31 D35 D38 D39 D42 D45 D47 D53 D55 D60 D61 } \\
\text { D62 D63 D64 D65 D66 D67 D68 D69 D70 }\end{array}$ \\
\hline $\mathrm{PCl}$ & P11 P32 P33 P34 P35 P36 P37 P38 P40 P42 P43 P44 P47 P50 \\
\hline PC2 & P12 P13 P15 P16 P17 P18 P19 P20 P21 P22 P23 P25 P26 P27 P28 P30 P41 \\
\hline DC1 & D2 D3 D4 D5 D6 D7 D8 D9 D13 D14 D16 D17 D18 D19 D20 \\
\hline DC2 & D10 D11 D23 D24 D25 D26 D27 D29 D30 D32 D33 D34 D36 D37 D40 \\
\hline DC3 & D41 D43 D44 D46 D48 D49 D50 D51 D52 D54 D56 D57 D58 D59 \\
\hline
\end{tabular}

TABLE 5: Customer units' assignment in the grand coalition.

\begin{tabular}{|c|c|}
\hline & Customer units allocation \\
\hline \multicolumn{2}{|l|}{ LC } \\
\hline $\mathrm{LC}_{\mathrm{P}}$ & P1 P2 P3 P4 P6 P13 P14 P17 P22 P23 P25 P28 P30 P34 \\
\hline $\mathrm{LC}_{\mathrm{D}}$ & $\begin{array}{c}\text { D3 D6 D18 D21 D22 D23 D28 D36 D39 D45 D51 D52 D54 D59 D60 D61 D62 } \\
\text { D65 D66 D67 D68 D70 }\end{array}$ \\
\hline $\mathrm{PCl}$ & $\begin{array}{c}\text { P18 P19 P24 P26 P29 P31 P32 P35 P36 P37 P38 P39 P40 P42 P43 P44 P45 P46 P47 } \\
\text { P48 P49 P50 }\end{array}$ \\
\hline PC2 & P5 P7 P8 P9 P10 P11 P12 P15 P16 P20 P21 P27 P33 P41 \\
\hline $\mathrm{DCl}$ & $\begin{array}{l}\text { D1 D2 D4 D5 D7 D10 D11 D12 D13 D14 D15 D16 D19 D20 D25 D26 D38 D40 } \\
\text { D56 D57 D58 D63 D64 D47 }\end{array}$ \\
\hline DC2 & D8 D9 D24 D27 D29 D33 D34 D35 D37 \\
\hline DC3 & D17 D30 D31 D32 D41 D42 D43 D44 D46 D48 D49 D50 D53 D55 D69 \\
\hline
\end{tabular}


TABLE 6: Algorithms optimization results comparison.

\begin{tabular}{|c|c|c|c|c|c|c|c|c|}
\hline \multirow{2}{*}{ Sequence } & \multicolumn{4}{|c|}{ Total cost (dollar) } & \multicolumn{4}{|c|}{ Number of iterations } \\
\hline & GA-PSO & CPTS & HGA & TLBO & GA-PSO & CPTS & HGA & TLBO \\
\hline 1 & 16971 & 17632 & 18639 & 17451 & 487 & 535 & 495 & 572 \\
\hline 2 & 17235 & 17715 & 18334 & 17237 & 451 & 541 & 516 & 593 \\
\hline 3 & 17158 & 17543 & 17865 & 17382 & 463 & 559 & 531 & 604 \\
\hline 4 & 16971 & 17687 & 18026 & 17237 & 504 & 528 & 519 & 615 \\
\hline 5 & 16971 & 17819 & 17865 & 17469 & 476 & 556 & 534 & 577 \\
\hline 6 & 17023 & 17543 & 18223 & 17520 & 479 & 545 & 522 & 565 \\
\hline 7 & 16971 & 17726 & 18409 & 17334 & 495 & 537 & 503 & 599 \\
\hline 8 & 16971 & 17678 & 17987 & 17237 & 510 & 533 & 535 & 611 \\
\hline 9 & 16971 & 17799 & 18172 & 17256 & 503 & 529 & 511 & 600 \\
\hline 10 & 17196 & 17841 & 18464 & 17321 & 473 & 545 & 496 & 587 \\
\hline 11 & 16971 & 17543 & 18326 & 17292 & 492 & 552 & 507 & 591 \\
\hline 12 & 17304 & 17764 & 17865 & 17438 & 455 & 527 & 543 & 587 \\
\hline 13 & 16971 & 17815 & 18079 & 17513 & 507 & 560 & 529 & 572 \\
\hline 14 & 17189 & 17686 & 17897 & 17237 & 481 & 521 & 535 & 607 \\
\hline 15 & 16971 & 17752 & 18363 & 17543 & 497 & 519 & 518 & 556 \\
\hline 16 & 16971 & 17543 & 17865 & 17364 & 481 & 548 & 550 & 597 \\
\hline 17 & 17105 & 17690 & 18144 & 17419 & 473 & 525 & 532 & 592 \\
\hline 18 & 16971 & 17778 & 18251 & 17292 & 499 & 536 & 517 & 621 \\
\hline 19 & 16971 & 17639 & 17962 & 17237 & 512 & 552 & 523 & 606 \\
\hline 20 & 17113 & 17617 & 17994 & 17351 & 477 & 527 & 510 & 575 \\
\hline Average & 17049 & 17691 & 18137 & 17357 & 486 & 539 & 521 & 591 \\
\hline$t$-test & & -20.26 & -17.79 & -8.67 & & -10.73 & -6.34 & -21.31 \\
\hline$p$ value & & $2.5 E-14$ & $2.7 E-13$ & $5 E-08$ & & $1.7 E-9$ & $4.4 E-6$ & $1 E-14$ \\
\hline
\end{tabular}

heuristic methods [9]. TLBO considers the solutions proposed by individuals' search, two-phase optimization process including teacher and learner phases to obtain the global best fitness values (Dokeroglu, 2015). Each algorithm is executed 20 times, and the best solution is selected as the optimal cost for convergence. The optimal total cost and the number of iterations can be used to measure the effectiveness of four algorithms shown in Table 6.

As shown in Table 6, the $t$-test and $p$ value indicate that the total cost and the number of iterations are significantly different with the three algorithms. In addition, the number of times GA-PSO hybrid algorithm finds out the optimal solution is higher. For example, the best solution appears 12 out of 20 times with the GA-PSO, while it only appears four or five times with CPTS, HGA, and TLBO.

Compared with other three algorithms, the average optimization cost and number of iterations from our proposed algorithm are of better quality. Thus, we can conclude that GA-PSO has the following merits: (1) exchanging best-fit chromosomes and worst-fit particles between the GA and the PSO algorithm enhances the GA-PSO's capability to obtain better solutions. For example, the GA-PSO algorithm has a higher probability to obtain the best solution. (2) The proposed algorithm combines GA and PSO algorithm's global and local search capability. As illustration, the average cost from the proposed hybrid algorithm is lower than that of CPTS, HGA, and TLBO algorithms by $3.8 \%, 6.4 \%$, and $1.8 \%$, respectively. (3) The termination and periodic iteration rules are reasonably designed in the GA-PSO hybrid algorithm procedure, which improves the efficiency of the hybrid algorithm. Therefore, the average number of iterations from GA-PSO is significantly less than that of the CPTS, HGA, and TLBO algorithms by 53, 35, and 105 iterations, respectively. These findings indicate that our hybrid algorithm has better capability to obtain the optimal solution than that of the other three algorithms.

6.3. MCRS Model Application and Coalition Sequence Selection. Previous studies have already shown the efficiency of MCRS at profit distribution in multiple players' games [28]. Indeed, this method can be used to incorporate the coordinator's payoff into the design of the collective profit allocation plan and guarantee the stability of coalitions. Based on the fact that our case study's coordinator (LC) is also part of the network and receives the highest incentive from the government, we assume there is no extra payoff and set the synergy requirement $\sigma=0$. In this study, we first consider the formation of a grand coalition before splitting the group into two subcoalitions and evaluate the influences of each of these scenarios. The combined form of all nonempty alliances is shown in Table 7.

The sum of the revenue of $\mathrm{LC}_{\mathrm{P}}$ and $\mathrm{LC}_{\mathrm{D}}$ constitute the total profit of LC. Profit allocation results display differences in facilities' benefits as they appear in different coalitions. For example, PCl's initial profit while being alone is 1158, but, after 
TABLE 7: Profit allocation in two-echelon logistics distribution network.

\begin{tabular}{|c|c|c|}
\hline$S$ & $V(t)$ & $\varphi(s, v)$ \\
\hline $\mathrm{LC}_{\mathrm{P}}$ & 648 & $(648 ; \ldots ; \ldots ; \ldots ; \ldots ; \ldots ; \ldots)$ \\
\hline $\mathrm{LC}_{\mathrm{D}}$ & 1422 & $(1422 ; \ldots ; \ldots ; \ldots ; \ldots ; \ldots ; \ldots)$ \\
\hline PC1 & 1158 & $(\ldots ; 1158 ; \ldots ; \ldots ; \ldots ; \ldots ; \ldots)$ \\
\hline PC2 & 688 & $(\ldots ; \ldots ; 688 ; \ldots ; \ldots ; \ldots ; \ldots)$ \\
\hline $\mathrm{DC} 1$ & 1239 & $(\ldots ; \ldots ; \ldots ; \ldots ; 1239 ; \ldots ; \ldots)$ \\
\hline DC2 & 769 & $(\ldots ; \ldots ; \ldots ; \ldots ; \ldots ; 769 ; \ldots)$ \\
\hline DC3 & 760 & $(\ldots ; \ldots ; \ldots ; \ldots ; \ldots ; \ldots ; 760)$ \\
\hline $\mathrm{LC}_{\mathrm{P}}, \mathrm{PC1}$ & 2353 & $(922 ; 1431 ; \ldots ; \ldots ; \ldots ; \ldots ; \ldots)$ \\
\hline $\mathrm{LC}_{\mathrm{P}}, \mathrm{PC} 2$ & 2796 & $(1378 ; \ldots ; 1418 ; \ldots ; \ldots ; \ldots ; \ldots)$ \\
\hline $\mathrm{LC}_{\mathrm{D}}, \mathrm{DC} 1$ & 3050 & $(\ldots ; \ldots ; \ldots ; 1617 ; 1433 ; \ldots ; \ldots)$ \\
\hline $\mathrm{LC}_{\mathrm{D}}, \mathrm{DC} 2$ & 2824 & $(\ldots ; \ldots ; \ldots ; 1738 ; \ldots ; 1086 ; \ldots)$ \\
\hline $\mathrm{LC}_{\mathrm{D}}, \mathrm{DC} 3$ & 2886 & $(\ldots ; \ldots ; \ldots ; 1774 ; \ldots ; \ldots ; 1112)$ \\
\hline $\mathrm{LC}_{\mathrm{P}}, \mathrm{PC} 1, \mathrm{PC} 2$ & 4411 & $(1377 ; 1616 ; 1418 ; \ldots ; \ldots ; \ldots ; \ldots)$ \\
\hline $\mathrm{LC}_{\mathrm{D}}, \mathrm{DC} 1, \mathrm{DC} 2$ & 3308 & $(\ldots ; \ldots ; \ldots ; 1372 ; 1212 ; 724 ; \ldots)$ \\
\hline $\mathrm{LC}_{\mathrm{D}}, \mathrm{DC} 1, \mathrm{DC} 3$ & 4901 & $(\ldots ; \ldots ; \ldots ; 2121 ; 1564 ; \ldots ; 1216)$ \\
\hline $\mathrm{LC}_{\mathrm{D}}, \mathrm{DC} 2, \mathrm{DC} 3$ & 5310 & $(\ldots ; \ldots ; \ldots ; 2203 ; \ldots ; 1769 ; \ldots ; 1338)$ \\
\hline $\mathrm{LC}_{\mathrm{P}}, \mathrm{PC} 1, \mathrm{PC} 2, \mathrm{LC}_{\mathrm{D}}, \mathrm{DC} 1$ & 7644 & $(1438 ; 1677 ; 1478 ; 1617 ; 1434 ; \ldots ; \ldots)$ \\
\hline $\mathrm{LC}_{\mathrm{P}}, \mathrm{PC} 1, \mathrm{PC} 2, \mathrm{LC}_{\mathrm{D}}, \mathrm{DC} 2$ & 7404 & $(1434 ; 1673 ; 1474 ; 1738 ; \ldots ; 1085 ; \ldots)$ \\
\hline $\mathrm{LC}_{\mathrm{P}}, \mathrm{PC} 1, \mathrm{PC} 2, \mathrm{LC}_{\mathrm{D}}, \mathrm{DC} 3$ & 7470 & $(1435 ; 1674 ; 1475 ; 1774 ; \ldots ; \ldots ; 1112)$ \\
\hline $\mathrm{LC}_{\mathrm{P}}, \mathrm{PC} 1, \mathrm{PC} 2, \mathrm{LC}_{\mathrm{D}}, \mathrm{DC} 1, \mathrm{DC} 2$ & 7819 & $(1418 ; 1653 ; 1439 ; 1372 ; 1212 ; 724 ; \ldots)$ \\
\hline $\mathrm{LC}_{\mathrm{P}}, \mathrm{PC} 1, \mathrm{PC} 2, \mathrm{LC}_{\mathrm{D}}, \mathrm{DC} 1, \mathrm{DC} 3$ & 9509 & $(1440 ; 1687 ; 1481 ; 2121 ; 1564 ; \ldots ; 1216)$ \\
\hline $\mathrm{LC}_{\mathrm{P}}, \mathrm{PC} 1, \mathrm{PC} 2, \mathrm{LC}_{\mathrm{D}}, \mathrm{DC} 2, \mathrm{DC} 3$ & 9881 & $(1444 ; 1669 ; 1457 ; 2204 ; \ldots ; 1769 ; 1338)$ \\
\hline $\mathrm{LC}_{\mathrm{P}}, \mathrm{PC} 1, \mathrm{PC} 2, \mathrm{LC}_{\mathrm{D}}, \mathrm{DC} 1, \mathrm{DC} 2, \mathrm{DC} 3$ & 12392 & $(1455 ; 1694 ; 1495 ; 2527 ; 1662 ; 1502 ; 2057)$ \\
\hline
\end{tabular}

TABLE 8: SMP based grand coalition sequence.

\begin{tabular}{|c|c|c|c|c|c|c|c|}
\hline \multicolumn{8}{|c|}{$\pi_{1}=\left\{\mathrm{LC}_{\mathrm{P}}, \mathrm{PC} 1, \mathrm{PC} 2, \mathrm{LC}_{\mathrm{D}}, \mathrm{DC} 1, \mathrm{DC} 3, \mathrm{DC} 2\right\}$} \\
\hline Player $i$ & $\mathrm{LC}_{\mathrm{P}}$ & PC1 & PC2 & $\mathrm{LC}_{\mathrm{D}}$ & $\mathrm{DC} 1$ & DC3 & DC2 \\
\hline$\eta(i, \pi, 1)$ & $11.9 \%$ & & & & & & \\
\hline$\eta(i, \pi, 2)$ & $16.9 \%$ & $25.0 \%$ & & & & & \\
\hline$\eta(i, \pi, 3)$ & $26.3 \%$ & $29.2 \%$ & $32.0 \%$ & & & & \\
\hline$\eta(i, \pi, 4)$ & $26.3 \%$ & $29.2 \%$ & $32.0 \%$ & $28.5 \%$ & & & \\
\hline$\eta(i, \pi, 5)$ & $26.4 \%$ & $29.4 \%$ & $32.1 \%$ & $32.5 \%$ & $43.2 \%$ & & \\
\hline$\eta(i, \pi, 6)$ & $26.5 \%$ & $29.5 \%$ & $32.2 \%$ & $42.6 \%$ & $47.1 \%$ & $45.8 \%$ & \\
\hline$\eta(i, \pi, 7)$ & $26.7 \%$ & $29.6 \%$ & $32.4 \%$ & $51.0 \%$ & $51.1 \%$ & $50.1 \%$ & $77.5 \%$ \\
\hline
\end{tabular}

sharing customers and transportation resources with $\mathrm{LC}_{\mathrm{P}}$, $\mathrm{PCl}$ could save 1432 . However, in certain circumstances, the profit can decrease as another participant enters the coalition. DC2 can be considered as an example to the extent that its initial profit (769) decreases after joining $\mathrm{LC}_{\mathrm{D}}$ and $\mathrm{DC} 1$ and becomes 724 . In fact, rational decision making requires every participant to give priority to coalitions where the increase of benefits is guaranteed. Therefore, we can conclude that the stability of any coalition depends on the profit of the members before and after new members adhere. In the process to form the grand coalition, cost reduction percentages have been calculated, presented in Figure 5, and illustrated in Figure 6.

The logistics center is denoted by $\mathrm{LC}_{\mathrm{P}}$ and $\mathrm{LC}_{\mathrm{D}}$ in Figure 5, while the legend in Figure 6 refers to it as LCP and LCD. We can notice at the fourth step in Figure 6 the simultaneous entrance of LCD and DC1 to emphasize that the delivery capacities of LC should not be applicable unless there is a DC in the coalition.

The examination of coalition sequences is critical to the profit distribution strategy and to participants' willingness to become member. In other words, the order in which members are added to the coalition affects the distribution of profits and the satisfaction of SMP principles. Nevertheless, following the examination of every possible combination, the SMP based coalition sequence is $\pi_{1}=$ $\left\{\mathrm{LC}_{\mathrm{P}}, \mathrm{PC} 1, \mathrm{PC} 2, \mathrm{LC}_{\mathrm{D}}, \mathrm{DC} 1, \mathrm{DC} 3, \mathrm{DC} 2\right\}$. Table 8 shows the cost reduction matrix representing members' behaviors as a new facility enters $\pi_{1}$.

In the design of the grand coalition, we have considered $\mathrm{LC}_{\mathrm{P}}$ joining first the coalition followed by PC1 and PC2 in 


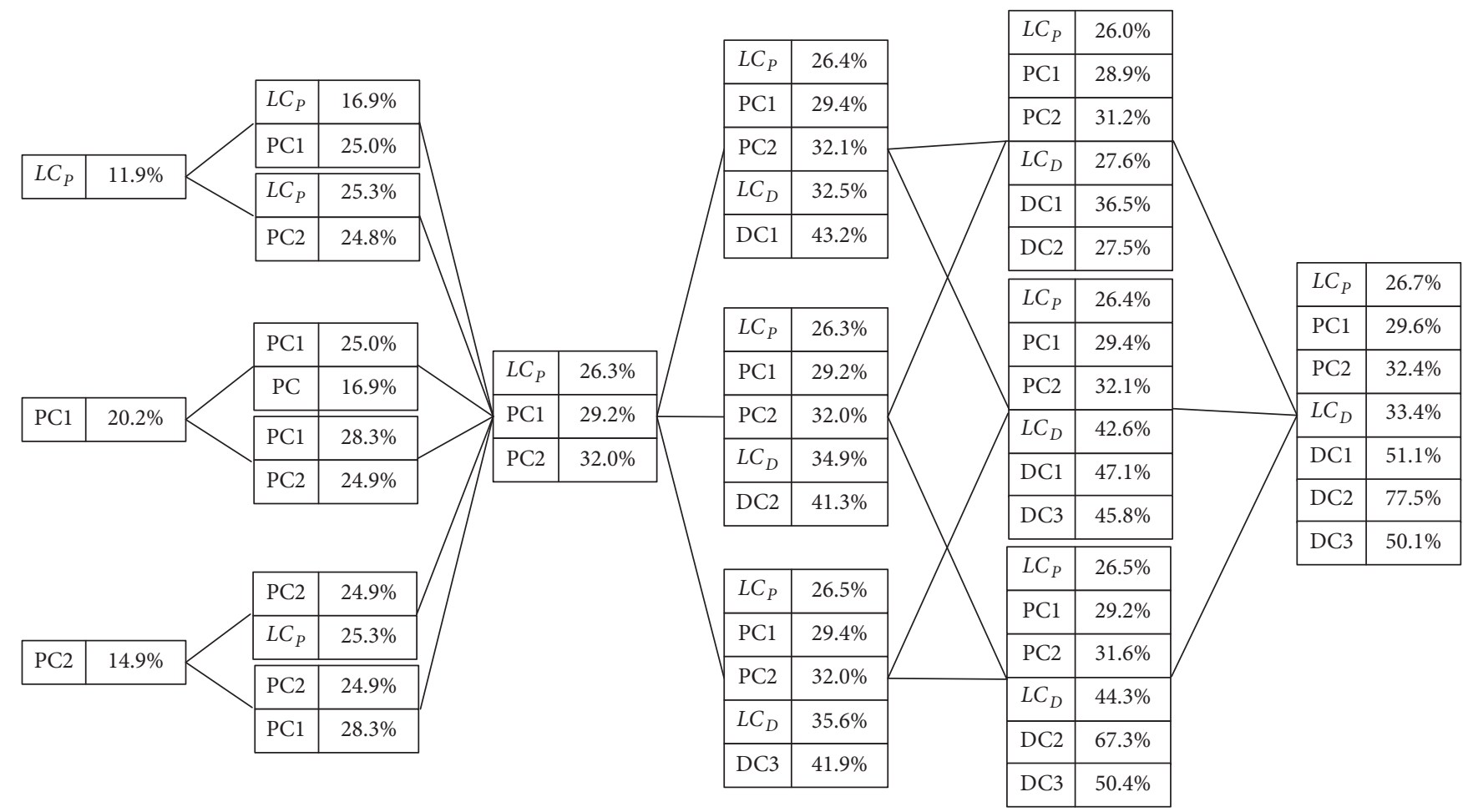

FIGURE 5: Grand alliance formation process.

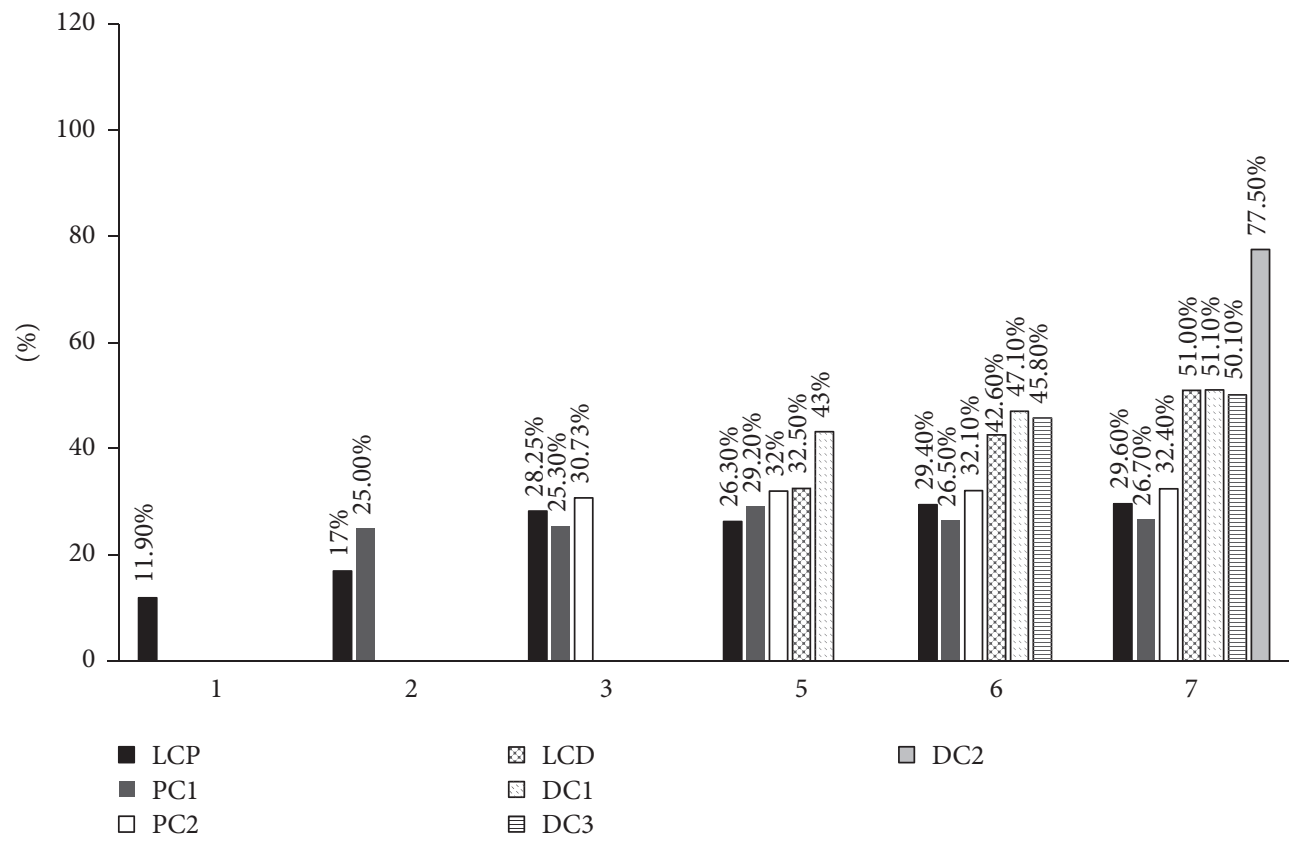

FIGURE 6: Cost reduction percentage diagram for the grand coalition.

order to assure that pickup activities are completed before deliveries and to conform to the proposed THCLN. The percentages of total operating costs reduced by LC, PCl, and PC2, at the level of unique pickup operations in the coalition, are, respectively, $26.3 \%, 29.2 \%$, and $32.0 \%$. After the adhesion of distribution centers in the order $\{\mathrm{DC} 1, \mathrm{DC} 3, \mathrm{DC} 2\}$, the final sequence for the grand coalition $\{\mathrm{LCP}, \mathrm{PC} 1, \mathrm{PC} 2, \mathrm{LCD}$, DC1, DC3, DC2 $\}$ yields as cost reduction percentage sequence $\{26.7 \%, 29.7 \%, 32.4 \%, 33.4 \%, 51.1 \%, 50.1 \%, 77.5 \%\}$, respectively. In the formation process, the profits of PCs have increased with the addition of DCs. In brief, we can conclude that LC as coordinator and the upper-level facility of PCs 
TABle 9: Profit allocations according to MCRS, Shapley, CGA, and GQP.

\begin{tabular}{lcccc}
\hline & MCRS & Shapley & CGA & GQP \\
\hline LC & 1377 & 1316 & 1344 & 1344 \\
PC1 & 1616 & 1554 & 1312 & 1312 \\
PC2 & 1417 & 1541 & 1755 & 1755 \\
LCD & 2527 & 2691 & 2923 & 2923 \\
DC1 & 1662 & 1572 & 805 & 805 \\
DC2 & 1502 & 1472 & 1214 & 1214 \\
DC3 & 2058 & 2013 & 2807 & 2807 \\
\hline
\end{tabular}

TABLE 10: Core center and distance comparison table.

\begin{tabular}{|c|c|c|c|}
\hline Method & Profit allocation & Core center & Distance \\
\hline MCRS & $\begin{array}{c}(1454.81,1693.83,1494.81,2527.37 \\
1661.90,1501.91,2057.65)\end{array}$ & \multirow{4}{*}{$\begin{array}{c}(1188.91,1695.29,1529.81,2328.50 \\
1581.68,1455.18,2130.29)\end{array}$} & 354.09 \\
\hline Shapley & $\begin{array}{c}(1315.98,1554.43,1540.91,2691.34 \\
1572.2,1472.27,2013.04)\end{array}$ & & 446.64 \\
\hline CGA & $\begin{array}{c}(1421.65,1389.64,1832.17,2923.34 \\
804.72,1214.17,2806.61)\end{array}$ & & 1308.43 \\
\hline GQP & $\begin{array}{c}(1421.65,1389.64,1832.17,2923.34 \\
804.72,1214.17,2806.61)\end{array}$ & & 1308.43 \\
\hline
\end{tabular}

and DCs can better optimize the entire logistics network. In this article, we required LC to be first member to enter the coalition. However in other circumstances, LC may not consider the value of its profit and allow another facility to make the first move for the sake of a more stable coalition.

6.4. Alliance Stability. In this section, we examine the accuracy of the MCRS method at providing the best profit distribution schemes in the investigated case. In this regard, three different methods are used to calculate appropriate profits of each facility $[29,30]$ : the Shapley value model, the Cost Gap Allocation (CGA) model, and the Game Quadratic Programming (GQP) method. Moreover, the Euclidean distance between each distribution scheme and core center of the grand coalition will be evaluated to verify each method's performance. In accordance with the snowball theory [31], the best strategy should be the closest to the center. The vector at the core is determined using formula (25) where the collective profit of the grand coalition is $V(Z), \beta$ represents a member and $\alpha$ is an analytic expression to control the scope of the core. In addition, Table 9 presents the results of the four methods, and Table 10 gives the core center and corresponding distances.

$$
\frac{v(Z)-v(Z-\{\beta\})}{v(Z)} \times \alpha+\sum_{k \in Z}^{k \neq i} y_{k}=v(Z-\{\beta\}) \text {. }
$$

Despite the allocation of lowest profits to PC2, LCD, and DC2, as shown in Table 9, MCRS yields the lowest distance (354) to the core center in Table 10. Therefore, we can notice that MCRS is the closest to the center and confirms our profit distribution scheme the most appropriate. Besides, findings allow us to conclude that, in a cooperative coalition, individual profit should not be the most important criteria.
Decision makers and coordinators have to observe the collective impacts of cooperation on the network.

6.5. Analysis of Two Heterogeneous Subcoalitions' Network. Segmentation is a strategic management approach used to control complex networks and consists in dividing the components into small parts. Some researchers have studied cooperation in logistics network optimization but concentrated on the design of multiple coalitions. Thus, in addition to the grand coalition, this paper has also considered other cases where the network would consist in two heterogeneous coalitions. In other words, PCs can independently cooperate with LC and a DC can form a coalition with LC and other DCs. It is important to notify that possibilities of constituting groups with different types of centers are numerous, but this article only evaluates circumstances where the same type of facility joins with LC in a single coalition. Our purpose is to examine the influence of multiple and single heterogeneous coalition in logistics network. Therefore, based on our solution methodology, the cost reduction percentages of possible sequences under two subcoalitions scenarios are shown in Tables 11 and 12.

Different from DCs coalitions where the best sequence can be directly selected, PCs coalition sequences should first be subject to the constraint of LC first entering. Indeed as Table 11 depicts, the cost reduction of $\pi_{1}=\left\{\mathrm{PC} 1, \mathrm{LC}_{\mathrm{P}}, \mathrm{PC} 2\right\}$ is higher than the second option. However, according to the assumption that LC makes the first move, we are constrained to select sequence $\pi_{1}=\left\{\mathrm{LC}_{\mathrm{P}}, \mathrm{PC} 1, \mathrm{PC} 2\right\}$. From Table 12, we can observe that cost reduction trend increases more rapidly under $\pi_{2}=\left\{\mathrm{LC}_{\mathrm{D}}, \mathrm{DC} 1, \mathrm{DC} 3, \mathrm{DC} 2\right\}$. Therefore, the logistics network with two heterogeneous subcoalitions should be designed such that the first subcoalition follows the order $\left\{L_{P}, P C 1, P C 2\right\}$, whereas the second respects the sequence 
TABLE 11: Pickup coalition sequences based on SMP.

\begin{tabular}{lccc}
\hline$\pi_{1}=\left\{\mathrm{LC}_{\mathrm{P}}, \mathrm{PC} 1, \mathrm{PC} 2\right\}$ & & & PC1 \\
\hline Player $i$ & $\mathrm{LC}_{\mathrm{P}}$ & & \\
$\eta(i, \pi, 1)$ & $11.9 \%$ & $25.0 \%$ & $\mathrm{PC} 2$ \\
$\eta(i, \pi, 2)$ & $16.9 \%$ & $29.2 \%$ & $32.0 \%$ \\
$\eta(i, \pi, 3)$ & $26.3 \%$ & & $\mathrm{LC}$ \\
\hline$\pi_{1}=\left\{\mathrm{PC} 1, \mathrm{LC}_{\mathrm{P}}, \mathrm{PC} 2\right\}$ & & & $\mathrm{PC} 2$ \\
\hline Player $i$ & $\mathrm{PC} 1$ & $16.9 \%$ & \\
$\eta(i, \pi, 1)$ & $20.2 \%$ & $26.3 \%$ & $32.0 \%$ \\
$\eta(i, \pi, 2)$ & $25.0 \%$ & & \\
$\eta(i, \pi, 3)$ & $29.2 \%$ & & \\
\hline
\end{tabular}

TABLE 12: Distribution coalition sequences based on SMP.

\begin{tabular}{|c|c|c|c|c|}
\hline \multicolumn{5}{|c|}{$\pi_{2}=\left\{L_{D}, D C 1, D C 3, D C 2\right\}$} \\
\hline Player $i$ & $\mathrm{LC}_{\mathrm{D}}$ & $\mathrm{DC1}$ & DC3 & DC2 \\
\hline$\eta(i, \pi, 1)$ & $28.5 \%$ & & & \\
\hline$\eta(i, \pi, 2)$ & $32.5 \%$ & $43.2 \%$ & & \\
\hline$\eta(i, \pi, 3)$ & $42.6 \%$ & $47.1 \%$ & $45.8 \%$ & \\
\hline$\eta(i, \pi, 4)$ & $51.0 \%$ & $51.1 \%$ & $50.1 \%$ & $77.5 \%$ \\
\hline \multicolumn{5}{|c|}{$\pi_{2}=\left\{\mathrm{LC}_{\mathrm{D}}, \mathrm{DC} 3, \mathrm{DC} 1, \mathrm{DC} 2\right\}$} \\
\hline Player $i$ & $\mathrm{LC}_{\mathrm{D}}$ & DC3 & DC1 & DC2 \\
\hline$\eta(i, \pi, 1)$ & $28.5 \%$ & & & \\
\hline$\eta(i, \pi, 2)$ & $35.6 \%$ & $41.9 \%$ & & \\
\hline$\eta(i, \pi, 3)$ & $42.6 \%$ & $47.1 \%$ & $45.8 \%$ & \\
\hline$\eta(i, \pi, 4)$ & $51.0 \%$ & $51.1 \%$ & $50.1 \%$ & $77.5 \%$ \\
\hline
\end{tabular}

$\left\{\mathrm{LC}_{\mathrm{D}}, \mathrm{DC} 1, \mathrm{DC} 3, \mathrm{DC} 2\right\}$. The cost reduction percentage diagram of two subcoalitions is shown in Figure 7.

The horizontal axes on the graphical illustrations of Figures 7 (a) and 7(b) represent the increase in participants' number, while the vertical axes mark the percentage of each facility. The amount of reduced cost monotonically increases after new members enter, thereby proving that the solutions bind the SMP rules. In the final appearance of the two subcoalitions, $\mathrm{LC}_{\mathrm{P}}$ first joins the pickup coalition with $11.9 \%$ cost reduction and then $\mathrm{PC} 1$ joins the coalition for $\mathrm{LC}_{\mathrm{P}}$ and PC1 to reduce up to $16.9 \%, 25 \%$ of their expenses in one working period; finally, PC2 joins, and the cost reductions of $\mathrm{LC}_{\mathrm{P}}, \mathrm{PC} 1$, and $\mathrm{PC} 2$ become $26.3 \%, 29.2 \%$, and $32 \%$, respectively. As for the delivery coalition, $\mathrm{LC}_{\mathrm{D}}$ first joins the coalition with $28.5 \%$ cost reduction, then $\mathrm{DCl}$ joins the coalition, and the cost reductions of $\mathrm{LC}_{\mathrm{D}}$ and $\mathrm{DCl}$ become $32.5 \%$ and $43.2 \%$ respectively; next, $\mathrm{DC} 3$ joins, and $\mathrm{LC}_{\mathrm{D}}, \mathrm{DC} 1$, and DC3 can reduce up to $42.6 \%, 47.1 \%$, and $45.8 \%$ of their expenses in one working period; finally, DC2 enters to yield as cost reductions of $\mathrm{LC}_{\mathrm{D}}, \mathrm{DC1}, \mathrm{DC} 2$, and DC3: $51.0 \%, 51.1 \%$, $50.1 \%$, and $77.5 \%$ respectively.

6.6. Discussion. The analysis of the profit allocation plans for the grand coalition and under two heterogeneous subcoalitions is a critical phase in the coordinator's decision making process. It offers the opportunity to evaluate existing alternatives and guarantees the reliability of the final decision as well as the sustainability of the cooperative logistics
TABLE 13: Comparison of different network scenarios.

\begin{tabular}{lcc}
\hline & Two subcoalitions & The grand coalition \\
\hline LCP & 1377 & 1455 \\
PC1 & 1616 & 1694 \\
PC2 & 1417 & 1495 \\
LCD & 2527 & 2527 \\
DC1 & 1662 & 1662 \\
DC2 & 1502 & 1502 \\
DC3 & 2058 & 2058 \\
Total & 12159 & 12393 \\
\hline
\end{tabular}

network. Each player's payoff is compared so as to estimate their best choices and conclude the most stable network. Table 13 presents the allocated profits of the five logistics facilities considered in the current case study.

As shown in Table 13, comparisons show that the profit received by each pickup centers is higher when they join the grand coalition. For example, each pickup facility can observe an increase of $\$ 78$ in profit if they opt for the formation of the grand coalition. Subsequently, LCP, PC1, and PC2 will, respectively, reduce their profit by $5.4 \%, 4.6 \%$, and $5.2 \%$ while moving from the grand coalition to form subcoalitions. Meanwhile, delivery centers would not constitute major problems to the final decision because their profits remain the same either in heterogeneous subcoalition or in the grand 


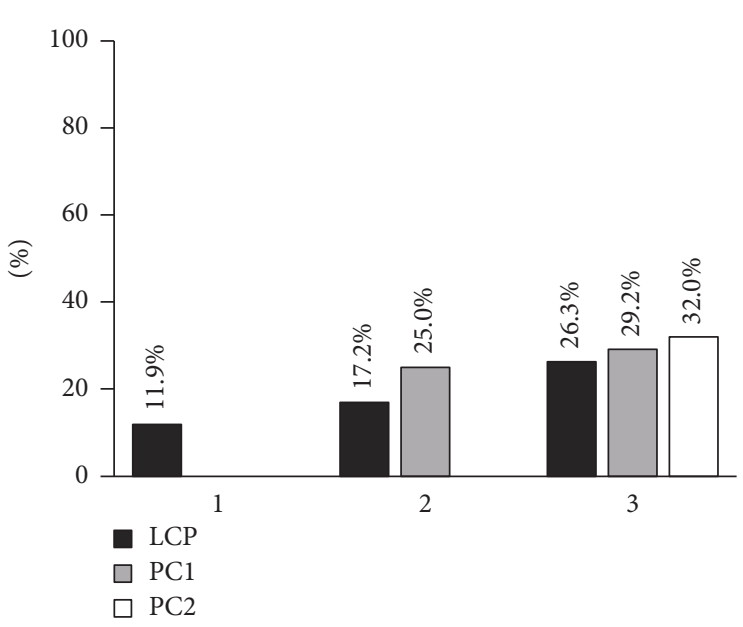

(a) First coalition formation

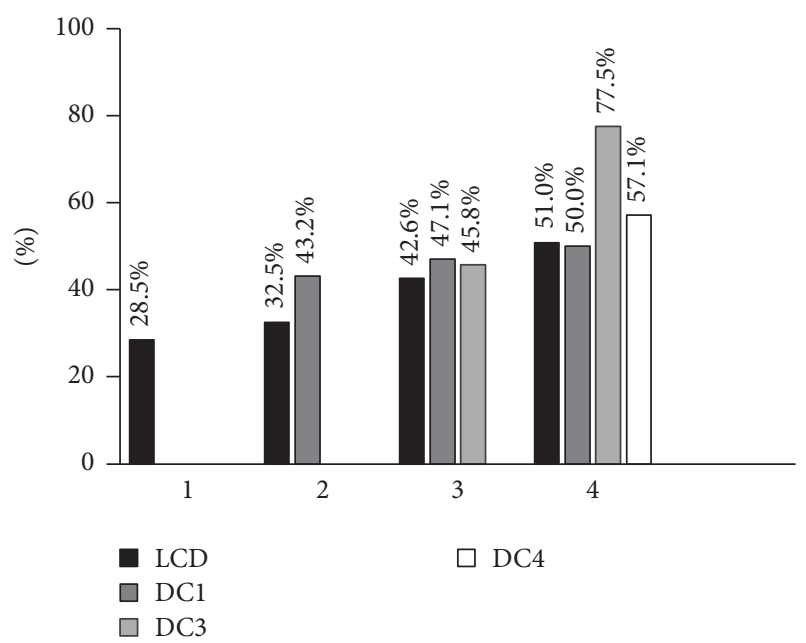

(b) Second coalition formation

FIGURE 7: Cost reduction percentage diagram for two subcoalitions.

coalition. Moreover, the collective profit in the logistics network increases when all the facilities join together to share resources. Though delivery centers' profits are identical, the difference generated by pickup activities in the collective network can enhance the superiority of the grand coalition. Therefore, findings indicate that forming a grand coalition rather than two subcoalitions is the best strategy to select both for participants and the coordinators.

In recent years, cooperation between logistics facilities has been playing an important role in the optimization of companies supply chains. Further incorporating transportation resources sharing can provide more cost savings. In addition, the existence of local governments' policies to encourage cooperation is also a sign of authorities will to achieve the sustainable development of their administrated areas. As one of the main development factors, transportation activities can be more organized with the reduction of the number of delivery and pickup vehicles. Therefore, encouraging the formation of a grand coalition is a relevant approach which would benefit not only logistics companies, but the whole society. However, forming a grand coalition may impose some managerial challenges. For example, the coalition's satisfaction of the strictly monotonic path principles may remain stable for a short period. Owing to the dynamics of modern logistics services and the variation of operations costs over time, participants can meet severe challenges resulting in internal financial and operational crisis. Consequently, the profit allocation will be influenced and the stability of the grand coalition threatened. As measure of prevention, different scenarios should be evaluated before coordinators launch negotiations. A possible means of handling such problems consists in splitting the grand coalition into several groups and evaluating potential risks related to individual facilities in the network before any final decision. Based on the proposed methodology, coordinators can also input estimated data for a decision period in order to find out the most appropriate type of coalition.

\section{Conclusions}

This paper studies the influence of cooperation among facilities on two echelons logistics networks with delivery and pickup operations. The grand and two heterogeneous coalitions have been studied to evaluate the preference of each participant willing to minimize expenses and maximize profit. The proposed THCLN is designed to interpret facilities possibilities of either cooperating with a similar center or participating in a group with different type of facilities. In other words, a pickup center can join another pickup center to form a subcoalition or join the grand coalition with distribution centers. The problem has been interpreted through a mixed integer linear model to optimize the total cost of nonempty coalitions. The solution methodology combines a GA-PSO hybrid algorithm to assign customers to the most suitable logistics facility and the MCRS method to properly allocate profits. Since different cooperative sequences have their corresponding cost reduction percentages, the selection of the best sequence is achieved using the SMP theory.

To test the effectiveness of THCLN as applicable network in real life, empirical analyses on a logistics network in Chongqing city, China, are conducted. As coordinator of the coalition formation process, we have selected the logistics center for its size and ability to collect and deliver goods. Comparisons have been done to prove that GA-PSO and MCRS are, respectively, proficient over other well-known methods. Computations have been carried out with CPTS, HGA, and TLBO algorithms, and GA-PSO has outperformed on the solution quality and the number of iterations. As for MCRS, its profit allocation scheme is closer to the core center than the Shapley value model, CGA, and GQP. Furthermore, we base on the assumption that the synergy requirement is equal to zero; that is, the coordinator should not receive an extra salary and share the collective profit to each member. The analysis of the results from the grand coalition and two heterogeneous subcoalitions concludes that forming a grand coalition is the most suitable. 
In the future, this research can be directed to optimize logistics networks of more than two echelons in order to expand the cooperative mechanism to a larger environment. Moreover, as customer assignment is a basic network optimization strategy, searching vehicle routing solutions can be another direction to implement the proposed approach. The impact of information sharing on the stability of the grand coalition can also be modeled and the variations in a coalition's lifetime can be simulated. Besides, the study of cooperative fresh food and medical supply chains can be done to experiment the relevance of our optimization methodology in other domains.

\section{Conflicts of Interest}

The authors declare that there are no conflicts of interest regarding the publication of this paper.

\section{Acknowledgments}

This research is supported by the National Natural Science Foundation of China (Projects nos. 71402011, 71301180, 71471024, 41401255, and 71432003), China Postdoctoral Science Foundation (Projects nos. 2017 T100692 and 2016M600735), National Social Science Foundation of Chongqing of China (no. 2017YBGL133), Natural Science Foundation of Chongqing of China (no. cstc2015jcyjA30012), and Postdoctoral Science Foundation of Sichuan Province, China (2017-22), and this research is partly supported by the Key Project of Human Social Science of Chongqing Municipal Education Commission (17SKG067 and 16SKGH067). In addition, the research is also partly supported by the Fundamental Research Funds for Central Universities (YWF-17-BJ-Y-04).

\section{References}

[1] O. Ö. Özener and Ö. Ergun, "Allocating costs in a collaborative transportation procurement network," Transportation Science, vol. 42, no. 2, pp. 146-165, 2008.

[2] Q. Wu, H. Ren, W. Gao, J. Ren, and C. Lao, "Profit allocation analysis among the distributed energy network participants based on Game-theory," Energy, vol. 118, pp. 783-794, 2017.

[3] H. Shi, Z. Li, W. Li, and Z. Yu, "Application of particle swarm optimization based on clustering analysis in logistics distribution," in Proceedings of the 2009 International Conference on Management of E-Commerce and E-Government, pp. 291-295, Nanchang, China, September 2009.

[4] R. Mesa-Arango and S. V. Ukkusuri, "Demand clustering in freight logistics networks," Transportation Research Part E: Logistics and Transportation Review, vol. 81, pp. 36-51, 2015.

[5] S. Baranowski, E. Busko, S. Shishlo et al., "Formation mechanism of logistics cluster in Belarus," Agriculture and Agricultural Science Procedia, vol. 7, pp. 12-20, 2015.

[6] I. Kumar, A. Zhalnin, A. Kim, and L. J. Beaulieu, "Transportation and logistics cluster competitive advantages in the U.S. regions: A cross-sectional and spatio-temporal analysis," Research in Transportation Economics, vol. 61, pp. 25-36, 2017.
[7] W.-J. Li and J. M. Smith, "An algorithm for quadratic assignment problems," European Journal of Operational Research, vol. 81, no. 1, pp. 205-216, 1995.

[8] I. Sghir, J.-K. Hao, I. B. Jaafar, and K. Ghédira, "A multi-agent based optimization method applied to the quadratic assignment problem," Expert Systems with Applications, vol. 42, no. 23, pp. 9252-9262, 2015.

[9] Z. Drezner, "Extensive experiments with hybrid genetic algorithms for the solution of the quadratic assignment problem," Computers \& Operations Research, vol. 35, no. 3, pp. 717-736, 2008.

[10] C. Chen, M. Li, J. Sui, K. Wei, and Q. Pei, "A genetic algorithmoptimized fuzzy logic controller to avoid rear-end collisions," Journal of Advanced Transportation, vol. 50, no. 8, pp. 17351753, 2016.

[11] Y. Shimizu and T. Miura, "Effect of topology on parallel computing for optimizing large scale logistics through binary PSO," Computer Aided Chemical Engineering, vol. 30, pp. 12471251, 2012.

[12] A. Čupić and D. Teodorović, "A multi-objective approach to the parcel express service delivery problem," Journal of Advanced Transportation, vol. 48, no. 7, pp. 701-720, 2014.

[13] B. Haddar, M. Khemakhem, S. Hanafi, and C. Wilbaut, "A hybrid heuristic for the 0-1 Knapsack Sharing Problem," Expert Systems with Applications, vol. 42, no. 10, pp. 4653-4666, 2015.

[14] J. Guo, X. Wang, S. Fan, and M. Gen, "Forward and reverse logistics network and route planning under the environment of low-carbon emissions: A case study of Shanghai fresh food E-commerce enterprises," Computers \& Industrial Engineering, vol. 106, pp. 351-360, 2017.

[15] Y. Wang, X. Ma, M. Liu et al., "Cooperation and profit allocation in two-echelon logistics joint distribution network optimization," Applied Soft Computing, vol. 56, pp. 143-157, 2017.

[16] Y. Wang, X. Ma, Z. Li, Y. Liu, M. Xu, and Y. Wang, "Profit distribution in collaborative multiple centers vehicle routing problem," Journal of Cleaner Production, vol. 144, pp. 203-219, 2017.

[17] A. Alshamsi and A. Diabat, "A genetic algorithm for reverse logistics network design: a case study from the GCC," Journal of Cleaner Production, vol. 151, pp. 652-669, 2017.

[18] A. Atashpendar, B. Dorronsoro, G. Danoy, and P. Bouvry, "A scalable parallel cooperative coevolutionary PSO algorithm for multi-objective optimization," Journal of Parallel and Distributed Computing, vol. 112, no. 2, pp. 111-125, 2018.

[19] B. Dai and H. Chen, "Profit allocation mechanisms for carrier collaboration in pickup and delivery service," Computers \& Industrial Engineering, vol. 62, no. 2, pp. 633-643, 2012.

[20] S. R. Dabbagh and M. K. Sheikh-El-Eslami, "Risk-based profit allocation to DERs integrated with a virtual power plant using cooperative Game theory," Electric Power Systems Research, vol. 121, pp. 368-378, 2015.

[21] S. Hernández and S. Peeta, "A carrier collaboration problem for less-than-truckload carriers: characteristics and carrier collaboration model," Transportmetrica A: Transport Science, vol. 10, no. 4, pp. 327-349, 2014.

[22] M. Guajardo and M. Rönnqvist, "Operations research models for coalition structure in collaborative logistics," European Journal of Operational Research, vol. 240, no. 1, pp. 147-159, 2015.

[23] Y. Wang, X. Ma, and Y. Lao, "A fuzzy-based customer clustering approach with hierarchical structure for logistics network optimization," Expert Systems with Applications, vol. 41, no. 2, pp. 521-534, 2014. 
[24] J. Kennedy and R. Eberhart, "Particle swarm optimization," in Proceedings of the IEEE International Conference on Neural Networks, pp. 1942-1948, Perth, Australia, December 1995.

[25] H. Chen, A. M. K. Cheng, and Y. Kuo, "Assigning real-time tasks to heterogeneous processors by applying ant colony optimization," Journal of Parallel and Distributed Computing, vol. 71, no. 1, pp. 132-142, 2011.

[26] T. J. Ai and V. Kachitvichyanukul, "A particle swarm optimization for the vehicle routing problem with simultaneous pickup and delivery," Computers \& Operations Research, vol. 36, no. 5, pp. 1693-1702, 2009.

[27] M. A. Mohammed, M. K. Abd Ghani, R. I. Hamed, S. A. Mostafa, M. S. Ahmad, and D. A. Ibrahim, "Solving vehicle routing problem by using improved genetic algorithm for optimal solution," Journal of Computational Science, vol. 21, pp. 255262, 2017.

[28] S. H. Tijs and T. S. Driessen, "Game theory and cost allocation problems," Management Science, vol. 32, no. 8, pp. 1015-1028, 1986.

[29] F. Cruijssen, P. Borm, H. Fleuren, and H. Hamers, "Supplierinitiated outsourcing: a methodology to exploit synergy in transportation," European Journal of Operational Research, vol. 207, no. 2, pp. 763-774, 2010.

[30] M. Frisk, M. Göthe-Lundgren, K. Jörnsten, and M. Rönnqvist, "Cost allocation in collaborative forest transportation," European Journal of Operational Research, vol. 205, no. 2, pp. 448458, 2010.

[31] S. Lozano, P. Moreno, B. Adenso-Díaz, and E. Algaba, "Cooperative game theory approach to allocating benefits of horizontal cooperation," European Journal of Operational Research, vol. 229, no. 2, pp. 444-452, 2013. 


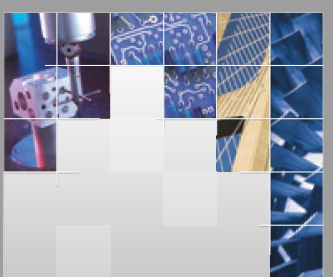

\section{Enfincering}
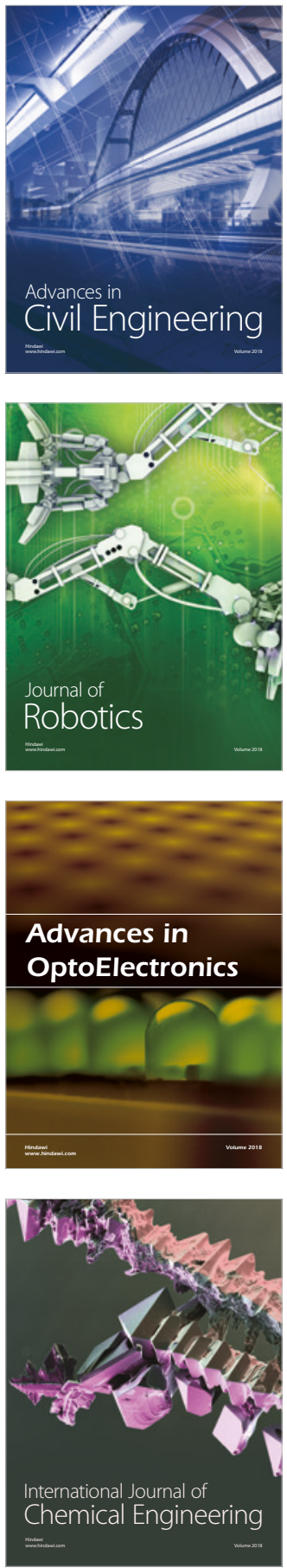

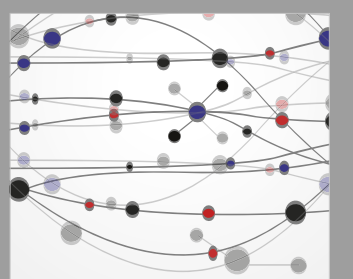

\section{Rotating \\ Machinery}

The Scientific World Journal

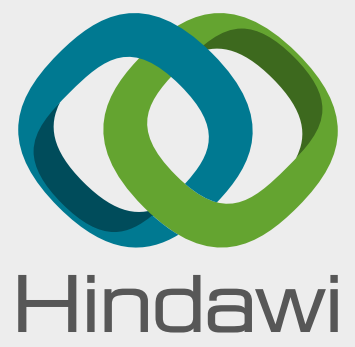

Submit your manuscripts at

www.hindawi.com
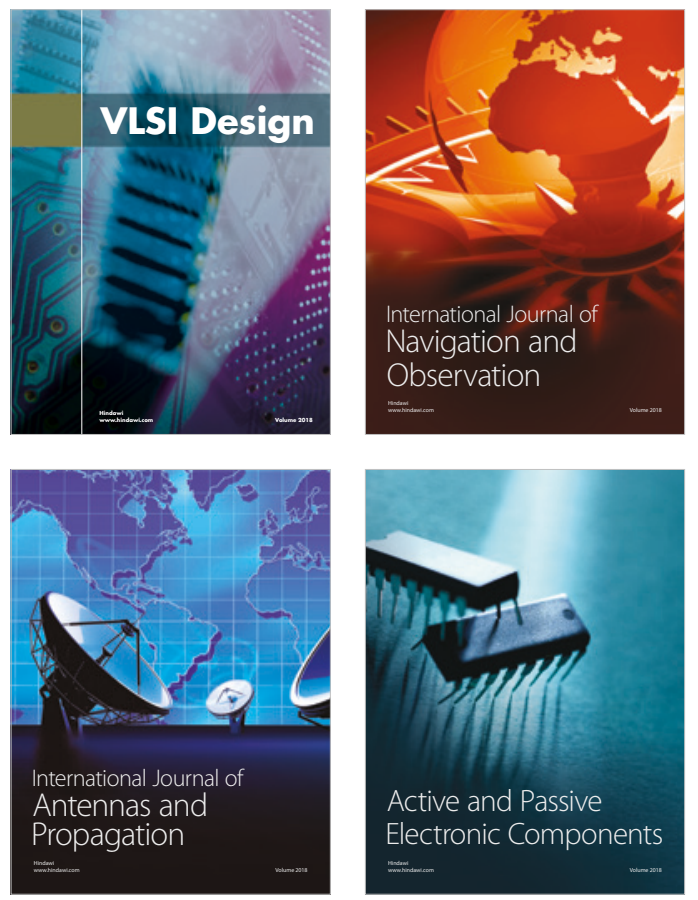
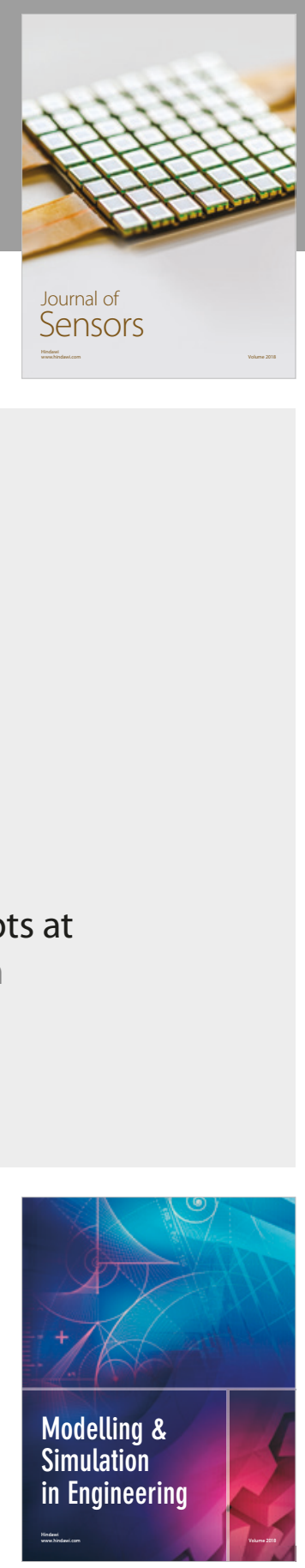

\section{Advances \\ Multimedia}
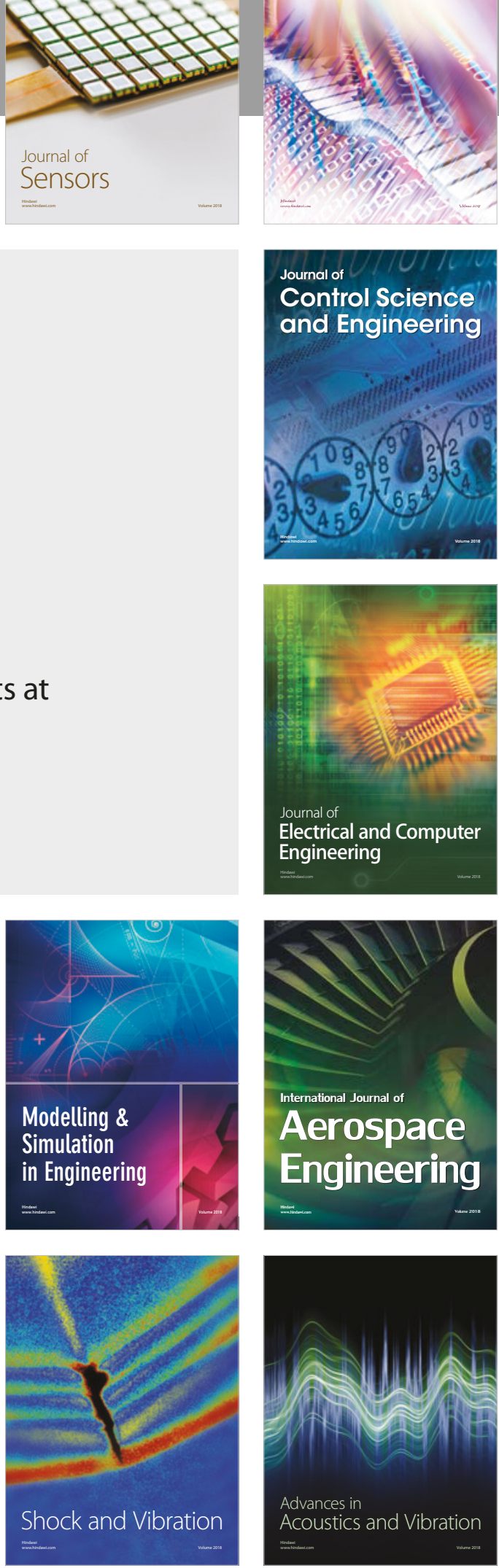